\title{
GENERALIZED MEAN CURVATURE FLOW IN CARNOT GROUPS
}

\author{
LUCA CAPOGNA AND GIOVANNA CITTI
}

\begin{abstract}
In this paper we study the generalized mean curvature flow of sets in the sub-Riemannian geometry of Carnot groups. We extend to our context the level sets method and the weak (viscosity) solutions introduced in the Euclidean setting in 29 and 14]. We establish two special cases of the comparison principle, existence, uniqueness and basic geometric properties of the flow.
\end{abstract}

\section{INTRODUCTION}

The evolution of hypersurfaces with normal velocity given by the mean curvature $K$ arises as the $L^{2}$ gradient flow of the Riemannian perimeter functional. A detailed list of references concerning the study of the mean curvature flow can be found in the monographs [25] and 67.

Although the mean curvature flow is locally smoothing, even starting with a smooth manifold as initial data, its flow may develop singularities before the extinction time, as in the famous example of the dumbbell in [29]. Several methods have been suggested in order to study the behavior of the flow past the formation of singularities: the method of currents introduced by Brakke [11, the method of generalized (viscosity) solutions indipendently developed by Chen, Giga and Goto [14], and by Evans and Spruck [29], [26], [27], [28], (see also the generalization by Ishii and Souganidis [44), De Giorgi's method of barriers [23] (which was studied in detail by Bellettini and Novaga [6], [7]) and the closely related definition by Barles and Souganidis [4] and [3].

Most pertinent to the present paper is the work in [29] where, following [55] the authors study the flow of level sets $M_{t}=\left\{x \in \mathbb{R}^{n} \mid u(x, t)=\right.$ $0\}$ where the function $u$ is a generalized solution of the degenerate

Key words and phrases. mean curvature flow, sub-Riemannian geometry, Carnot groups

The authors are partially funded by NSF Career grant DMS-0124318 (LC) and GALA project (GC). 
quasilinear, non-divergence form PDE

$$
\partial_{t} u(x, t)=K|\nabla u|=\sum_{i, j=1}^{n}\left(\delta_{i j}-\frac{\partial_{x_{i}} u \partial_{x_{j}} u}{|\nabla u|^{2}}\right) \partial_{x_{i} x_{j}}^{2} u
$$

Note that the PDE becomes degenerate exactly at the singularity points of the level sets, that is where $|\nabla u|$ vanishes. The level set approach was extended by Ilmanen [42]) to include the study of generalized flow of subsets in Riemannian manifolds.

The Riemannian mean curvature flow has been used both as a model for the study of sharp-interfaces in material science and in digital image processing. Recently the first layer of the mamalian visual cortex has been modelled as a smooth surface with a sub-Riemannian geometry ([40] and [58]). In this setting some perceptual phenomena such as the formation of subjective surfaces, are described as sub-Riemannian mean curvature flows and minimal surfaces (see [57], [17], 61], 38], and [37]).

The focus of the present paper is to study a model case of the subRiemannian analogue of the mean curvature flow: the horizontal mean curvature flow in Carnot groups.

Sub-Riemannian geometry is an extension of Riemannian geometry in which, given a manifold $G$, a metric $g_{0}$ is only prescribed on a subbundle $H G \subset T G$ (called horizontal bundle). The horizontal bundle is supposed to have the so-called bracket generating properties, i.e. there is a number $r \in \mathbb{N}$ such that all sections of $T G$ are generated by linear combinations of sections of $H G$ and their commutators up to order $r$. In a standard fashion one can associate a control distance $d_{C}$ (the CarnotCarathéodory distance) to the sub-Riemannian structure $\left(G, H G, g_{0}\right)$. Blow-up of such geometric structures (see [34], [50] and [60]) give rise to "linear" sub-Riemannian manifolds called Carnot groups (nilpotent Lie groups endowed with a control metric, see the next section for a precise definition). In a sense, Carnot groups are the model for the tangent spaces to sub-Riemannian manifolds.

Sub-Riemannan structures can also be seen as degenerate limit of Riemannian structures: Fix an orthonormal frame $\mathcal{F}_{0}=\left\{X_{1}, \ldots, X_{m}\right\}$ of sections of $H G$ and extend it to a frame $\mathcal{F}$ of $T G$. Define Riemannian metrics $g_{\epsilon}, \epsilon>0$, extensions of $g_{0}$ to all of $T G$, such that at each point, the length of any non-horizontal section in $\mathcal{F}$ is $\epsilon^{-1}$. If we denote by $d_{\epsilon}$ the distance function associated to $g_{\epsilon}$ then $\left(G, d_{\epsilon}\right) \rightarrow\left(G, d_{C}\right)$ in the sense of the Gromov-Hausdorff convergence between metric spaces 
(see [35], 34], and [51]). For a more in-depth presentation of SubRiemannian geometry we refer the reader to [64], [35], 34], [51], [12] and references therein.

If $G$ is a Carnot group and $M \subset G$ is a smooth hypersurface we define $\Sigma(M)$ the set of characteristic points of $M$, i.e. the points $x \in M$ where the horizontal structure is contained in the tangent space. Derridij [24] proved that $\Sigma(M)$ has zero surface measure, this result was later refined in [1] and [48]. Outside $\Sigma(M)$ one can define a horizontal normal $\boldsymbol{n}^{\mathbf{0}}$, the normalized projection onto $H M$ of the Riemannian normal (in any of the metrics $g_{\epsilon}$ ). Accordingly, the horizontal mean curvature $K_{0}$ can be defined as the first variation of the sub-Riemannian perimeter in the horizontal normal direction (see [21, [10], [59], [39] and [62]) 1 . Generically such curvature is unbounded in a neighborhood of $\Sigma(M)$ and cannot be defined at characteristic points.

The horizontal mean curvature flow of a hypersurface of a Carnot group $G$ is the flow $t \rightarrow M_{t} \subset G$ in which each point $x(t) \notin \Sigma\left(M_{t}\right)$ in the evolving manifold moves along the horizontal normal with speed given by the horizontal mean curvature. The corresponding equation, outside the characteristic set, is

$$
\frac{d x}{d t}=-K_{0} \boldsymbol{n}^{\mathbf{0}}
$$

Extending the techniques in [29], the evolving surface $M_{t}$ can be represented as zero level set of a function $u(x, t)$ which solves the PDE,

$$
\partial_{t} u(x, t)=\sum_{i, j=1}^{m}\left(\delta_{i j}-\frac{X_{i} u X_{j} u}{\sum_{i=1}^{m}\left(X_{i} u\right)^{2}}\right) X_{i} X_{j} u .
$$

There is an obvious immediate difficulty in the study of this equation: It is not well defined in $\Sigma\left(M_{t}\right)$. In contrast with the Euclidean setting the PDE becomes degenerate not only at singularities of the level sets, where the full (spatial) gradient of the solution $\nabla u(\cdot, t)$ vanishes, but also at characteristic points. It is in fact only the vanishing of the horizontal portion of the full gradient which determines the characteristic set. In a sense, points in $\Sigma\left(M_{t}\right)$ correspond to metric singularities in the set $M_{t}$.

In view of this new difficulty, in the present paper while we are able to prove existence for the general flow described in (1.3), at the moment we can prove comparison principles and uniqueness only for a special

\footnotetext{
${ }^{1}$ Closely linked to the study of mean curvature flow, the analysis of minimal surfaces in the sub-Riemannian setting has recently seen great activity [32, [56, [15, [16], 33, 22], 5] and [54).
} 
class of flows, i.e. either in the presence of particular classes of initial data or for graphs over Carnot groups.

Bonk and the first named author study in [10] some properties of smooth solutions of this equation. In that paper the solution is interpreted in the vanishing viscosity sense, i.e. limit of Riemannian mean curvature flows. However they assume the existence of such smooth vanishing viscosity solution.

For elliptic or parabolic PDE the notion of vanishing viscosity is equivalent to the notion of viscosity solution (see [20, Section 6]). This question has not yet been addressed in the sub-Riemannian setting, where however a number of authors have studied viscosity solutions for non degenerate PDE: 8], 9], 65], 66], 47], 2], and [49.

In the present paper we give a new definition of continuous (non smooth) viscosity solutions to (1.3). The novelty of our definition comes from the fact that the equation is totally degenerate at characteristic points, where the right hand side of (1.3) is not defined. While we cannot prove that such viscosity solutions are equivalent to vanishing viscosity solutions we establish existence and uniqueness of Lipschitz vanishing viscosity solutions for the initial value problem, and some basic geometric properties of the flow. Hence our results can be interpreted as special limit cases of Ilmanen's work [42] in the approximation $g_{\epsilon} \rightarrow g_{0}$ described above.

The paper is organized as follows: In section 2 we give the definition of viscosity solutions to (1.3) and recall some existing results. In section 3 we prove two particular cases of the comparison theorem between a bounded subsolution $u$ and a supersolution $v$ of equation (1.3), from which uniqueness follows. In order to do so it is quite standard to use as test function the difference of regularized versions of these two functions (the so called sup inf- convolutions) in two different points, with a penalization term. The choice of this test function and in particular of the penalization term depends crucially on the sub-Riemannian character of the problem. In Section 4 we identify simple classes of solutions (self-shrinking cylinders and stationary planes) and construct bounded barriers which will be used in the proof of existence and in the study of geometric properties of the flows. The construction of explicit solutions is not trivial in our setting. The metric sphere, which is self-shrinking and heavily used in the Euclidean setting (see [29]), does not have a self-similar evolution in our setting. Indeed it is an open question wether there is any closed manifold which gives rise to a self-similar solution. See [10] for a study of self-similar solutions in the Heisenberg group. 
In Section 5 we prove the existence of Lipschitz vanishing viscosity solutions, and the fact that they are also viscosity solutions. In the proof of existence we first provide higher order a priori estimates for the solutions of the approximating Riemannian flows. We cannot rely on the estimates proved by Ilmanen in [42] as they are dependent on curvature bounds, which fail in our setting. Moreover the noncommutativity of the vector fields $X_{i}$ makes it hard to prove a-priori higher order estimates. We deal with this problem by using both leftinvariant and right-invariant derivatives. Indeed, such left and right derivatives commute (by definition), allowing to easily differentiate the equation. This, along with a parabolic maximum principle yields the desired bounds.

In Section 6 we prove some simple geometric properties of the evolution. Lacking a complete comparison principle we cannot show that the generalized flow does not depend on the choice of the initial defining function, but only its zero level set. We show that if two sets $M, \hat{M}$ satisfy $M \subset \hat{M}$, then the inclusion $M_{t} \subset \hat{M}_{t}$ between their evolutions $M_{t}$, $\hat{M}_{t}$ persists for all times. Since this result depends on the comparison principle we need some additional hypothesis on $\hat{M}_{t}$.

For generalized level sets arising out of vanishing viscosity solutions, we show also that the right invariant control distance between two disjoint initial sets increases in the evolution. As a corollary we have that any initial compact set has a finite extinction time, i.e. the evolving set shrinks and eventually vanishes in a finite time.

To conclude, we recently learned that Dirr, Dragoni and Von Renesse have recently studied a probabilistic approach to the mean curvature flow in the context of the Heisenberg group in the same spirit of [63].

Acknowledgments. Part of the work on this paper was done while the authors were guests of the Centro di Ricerca Matematica Ennio de Giorgi, in Pisa, Italy. We thank the staff of the center, M. Giaquinta, F. Ricci and L. Ambrosio for their hospitality and for interesting conversations.

\section{Definitions AND PRELIminary Results}

2.1. Carnot group structure. Let $G$ be an analytic and simply connected Lie group with topological dimension $n$ and such that its Lie algebra $\mathcal{G}$ admits a stratification $\mathcal{G}=V^{1} \oplus V^{2} \oplus \ldots \oplus V^{r}$, where $\left[V^{1}, V^{j}\right]=V^{j+1}$, if $j=1, \ldots, r-1$, and $\left[V^{k}, V^{r}\right]=0, k=1, \ldots, r$. Such groups are called in [30, 31, and [64] stratified nilpotent Lie groups. Fix $X_{1}, \ldots, X_{m}$ a basis of $V^{1}$, called the horizontal frame, and complete it to a basis $\left(X_{1}, \ldots, X_{n}\right)$ of $\mathcal{G}$ by choosing for every $k=2, \cdots r$ a basis 
of $V_{k}$. If $X_{i}$ belongs to $V_{k}$, then we will set $d(i)=k$. We will denote by $x X=\sum_{i=1}^{n} x_{i} X_{i}$ a generic element of $\mathcal{G}$. Since the exponential map $\exp : \mathcal{G} \rightarrow G$ is a global diffeomorphism we use exponential coordinates in $G$, and denote $x=\left(x_{1}, \cdots, x_{n}\right)$ the point $\exp (x X)$. We also set $x_{H}=\left(x_{1}, \cdots, x_{m}\right)$ and $x_{V}=\left(x_{m+1}, \ldots, X_{n}\right)$ so that $x=\left(x_{H}, x_{V}\right)$. Define non-isotropic dilations as $\delta_{s}(x)=\left(s^{d(i)} x_{i}\right)$, for $s>0$.

We denote by $\left(X_{1}, \ldots, X_{n}\right)$ (resp. $\left.\left(\tilde{X}_{1}, \ldots, \tilde{X}_{n}\right)\right)$ the left invariant (resp. right invariant) translation of the frame $\left(X_{1}, \ldots, X_{n}\right)$ of $\mathcal{G}$. Set $H(0)=$ $V^{1}$, and for any $x \in G$ we let $H(x)=x H(0)=\operatorname{span}\left[X_{1}, \ldots, X_{m}\right](x)$. The distribution $x \rightarrow H(x)$ is called the horizontal sub-bundle $H$. On $H$ we define a left invariant positive definite form $g_{0}$, so that $X_{1} \cdots, X_{m}$ is an orthonormal frame. We let $\nabla=\left(X_{1}, \cdots, X_{m}\right)$ denote the horizontal gradient operator. The vectors $X_{1} \ldots X_{m}$ and their commutators span all the Lie algebra $\mathcal{G}$, and consequently verify Hörmander's finite rank condition ([41]). This allows to use the results from [53], and define a control distance $d_{C}(x, y)$ associated to the distribution $X_{1} \ldots X_{m}$, which is called the Carnot-Carathéodory metric (denote by $\tilde{d}_{C}$ the corresponding right invariant distance). We call the couple $\left(G, d_{C}\right)$ a Carnot Group.

We define a family of left invariant Riemannian metrics $g_{\epsilon}, \epsilon>0$ in $\mathcal{G}$ by requesting that $\left\{X_{1}, \cdots, X_{m} . \epsilon X_{m+1}, \cdots, \epsilon X_{n}\right\}$ is an orthonormal frame. We will denote by $d_{\epsilon}$ the corresponding distance functions. Correspondingly we use $\nabla_{\epsilon}$, (resp. $\tilde{\nabla}_{\epsilon}$ ) to denote the left (resp. right) invariant gradients.

It is well known'2 that $\left(G, d_{\epsilon}\right)$ converges in the Gromov-Hausdorff sense as $\epsilon \rightarrow 0$ to the sub-Riemannian space $\left(G, d_{C}\right)$. The CarnotCarathéodory metric is equivalent to a more explicitly defined pseudodistance function, that we will call (improperly) gauge distance, defined as

$$
|x|^{2 r !}=\sum_{k=1}^{r} \sum_{i=1}^{m_{k}}\left|x_{i, k}\right|^{\frac{2 r !}{k}}, \text { and } d(x, y)=\left|y^{-1} x\right|
$$

If $x \in G$ and $r>0$, we will denote by $B(x, r)=\{y \in G \mid d(x, y)<r\}$ the balls in the gauge distance.

We recall now the expression of the left invariant vector fields in exponential coordinates (see [60])

$$
X_{i}=\partial_{i}+\sum_{k=d(i)+1}^{r} \sum_{d(j)=k} p_{i k}^{j}(x) \partial_{j}
$$

\footnotetext{
${ }^{2}$ See for instance 35 .
} 
where $p_{i k}^{j}(x)$ is an homogeneous polynomial of degree $k-d(i)$ and depends only on $x_{h}$, with $d(1) \leq d(h) \leq k-d(i)$.

2.2. Horizontal mean curvature flow of hypersurfaces. Let $M \subset$ $G$ be a $C^{2}$ smooth hypersurface, denote by $\boldsymbol{n}^{\epsilon}$ the unit normal in the metric $g_{\epsilon}$ and by $\boldsymbol{n}^{\mathbf{0}}=\sum_{d(i)=1}\left(\boldsymbol{n}^{\mathbf{0}}\right)_{i} X_{i}$ its normalized projection in the $g_{\epsilon}$ norm onto the horizontal plane. Note that this is not dependent on $\epsilon$ and is well defined only outside the characteristic set $\Sigma(M)=\{x \in$ $\left.M \mid H(x) \subset T_{x} M\right\}$. The vector $\boldsymbol{n}^{\mathbf{0}}$ is called horizontal normal and its (horizontal) divergence

$$
K_{0}=\sum_{d(i)=1} X_{i} \boldsymbol{n}^{\mathbf{0}}
$$

is known as the horizontal mean curvature of $M$ at $x \notin \Sigma(M)$. Note that even for smooth (in the Euclidean sense) hypersurfaces the horizontal mean curvature may blow up near characteristic points.

We study the flow $t \rightarrow M_{t}$ where a point $x \in M_{t}$ evolves with velocity $\partial_{t} x=-K_{0} \boldsymbol{n}^{\mathbf{0}}$. The level set approach consists in studying a PDE describing the evolution of a function $u(x, t)$ such that ${ }^{3} M_{t}=$ $\{x \in G \mid u(x, t)=0\}$. In this setting one has $\boldsymbol{n}^{\epsilon}=\nabla_{\epsilon} u /\left|\nabla_{\epsilon} u\right|$ and $\boldsymbol{n}^{\mathbf{0}}=\nabla_{0} u /\left|\nabla_{0} u\right|$. Consequently, on a formal level, one has

$$
\begin{aligned}
\partial_{t} u(x(t), t) & =<\nabla_{0} u(x(t)), \partial_{t} x(t)>_{0}+\partial_{t} u(x, t) \\
& =-K_{0}<\nabla_{0} u, \boldsymbol{n}^{\mathbf{0}}>+\partial_{t} u=-K_{0}\left|\nabla_{0} u\right|+\partial_{t} u=0 .
\end{aligned}
$$

This problem is well approximated by the Riemannian mean curvature flows $\partial_{t} x=-K_{\epsilon} \boldsymbol{n}^{\boldsymbol{\epsilon}}$, where $K_{\epsilon}=\sum_{i=1}^{n} X_{i}^{\epsilon} \boldsymbol{n}_{i}{ }_{i}$ is the $g_{\epsilon}$ mean curvature of $M$. The corresponding evolution PDE for the level sets is $\partial_{t} u^{\epsilon}=K_{\epsilon}\left|\nabla_{\epsilon} u\right|$. We observe that for a given hypersuface, $\boldsymbol{n}^{\boldsymbol{\epsilon}} \rightarrow \boldsymbol{n}^{\mathbf{0}}$ and $K_{e} \rightarrow K_{0}$ as $\epsilon \rightarrow 0$, outside the characteristic set. We will prove in Section 5 that $u^{\epsilon} \rightarrow u$ weak solution of (2.3).

A simple computation shows that the mean curvature $K_{\epsilon}$ of the manifold $\{u(x)=0\}$ is given by the identity

$$
K_{\epsilon}\left|\nabla_{\epsilon} u\right|=\sum_{i, j=1}^{n}\left(\delta_{i j}-\frac{X_{i}^{\epsilon} u X_{j}^{\epsilon} u}{\left|\nabla_{\epsilon} u\right|^{2}}\right) X_{i}^{\epsilon} X_{j}^{\epsilon} u,
$$

\footnotetext{
${ }^{3}$ When a manifold is defined as a level set, we tacitly assume that the gradient of the defining function does not vanish in a neighborhood of the manifold.
} 
Outside of the characteristic set the horizontal mean curvature $K_{0}$ is expressed as

$$
K_{0}\left|\nabla_{0} u\right|=\sum_{i, j=1}^{m}\left(\delta_{i j}-\frac{X_{i} u X_{j} u}{\left|\nabla_{0} u\right|^{2}}\right) X_{i} X_{j} u
$$

Consequently (2.3) can be rewritten more explicitly as

$$
u_{t}=\sum_{i, j=1}^{m}\left(\delta_{i j}-\frac{X_{i} u X_{j} u}{\left|\nabla_{0} u\right|^{2}}\right) X_{i} X_{j} u, \text { for } x \in G, t>0 .
$$

If the Carnot group is a product $G=\tilde{G} \times \mathbb{R}$ and we use coordinates $(x, e) \in \tilde{G} \times \mathbb{R}$, then from (2.4) and by representing the function $u$ as $u(x, e, t)=e-U(x, t)$, we obtain a special class of evolutions, given by graphs over $\tilde{G}$ of the form $M_{t}=\{(x, U(x, t)) \mid x \in \tilde{G}, t>0\}$ where $U: \tilde{G} \rightarrow \mathbb{R}$ is a solution of

$$
U_{t}=\sum_{i, j=1}^{m}\left(\delta_{i j}-\frac{X_{i} U X_{j} U}{1+\left|\nabla_{0} U\right|^{2}}\right) X_{i} X_{j} U, \text { for } x \in \tilde{G}, t>0 .
$$

Note that such graphs are always non-characteristic.

2.2.1. Weak solutions. As in the Euclidean case, one cannot expect the smoothness of the solution to be preserved for all times. Moreover, even for smooth solutions, the horizontal gradient vanishes at all characteristic points making the equation degenerate. To overcome these difficulties we use the subelliptic analogue of viscosity solutions (see also for earlier related definitions [9], [66]).

Definition 2.1. A function $u \in C(G \times[0, \infty)$ is a weak subsolution of (2.4) in $G \times(0, \infty)$ if for any $(x, t) \in G \times(0, \infty)$ and any function $\phi \in C^{2}(G) \times(0, \infty)$ such that $u-\phi$ has a local maximum at $(x, t)$ then

$$
\partial_{t} \phi \leq \begin{cases}\sum_{i, j=1}^{m}\left(\delta_{i j}-\frac{X_{i} \phi X_{j} \phi}{\left|\nabla_{0} \phi\right|^{2}}\right) X_{i} X_{j} \phi & \text { if }\left|\nabla_{0} \phi\right| \neq 0 \\ \sum_{i, j=1}^{m}\left(\delta_{i j}-p_{i} p_{j}\right) X_{i} X_{j} \phi & \text { for some } p \in \mathbb{R}^{m},|p| \leq 1, \text { if }\left|\nabla_{0} \phi\right|=0 .\end{cases}
$$

A function $u \in C(G \times[0, \infty)$ is a weak supersolution of (2.4) if

$$
\partial_{t} \phi \geq \begin{cases}\sum_{i, j=1}^{m}\left(\delta_{i j}-\frac{X_{i} \phi X_{j} \phi}{\left|\nabla_{0} \phi\right|^{2}}\right) X_{i} X_{j} \phi & \text { if }\left|\nabla_{0} \phi\right| \neq 0 \\ \sum_{i, j=1}^{m}\left(\delta_{i j}-p_{i} p_{j}\right) X_{i} X_{j} \phi & \text { for some } p \in \mathbb{R}^{m},|p| \leq 1, \text { if }\left|\nabla_{0} \phi\right|=0 .\end{cases}
$$

A weak solution of (2.4) is a function $u$ which is both a weak subsolution and a weak supersolution. 
In the graph case $G=\tilde{G} \times \mathbb{R}$ when we consider only evolving surfaces of the form $M_{t}=\{e=U(x), x \in \tilde{G}\}$, we can also reduce the class of test functions in the previous definition to those of the form $\phi(e, x)=$ $e-\psi(x)$. In this way the definition of viscosity solutions becomes

Definition 2.2. A function $U \in C(\tilde{G} \times[0, \infty)$ is a weak subsolution of (2.5) in $\tilde{G} \times(0, \infty)$ if for any $(x, t) \in \tilde{G} \times(0, \infty)$ and any function $\psi \in C^{2}(\tilde{G}) \times(0, \infty)$ such that $U-\psi$ has a local maximum at $(x, t)$ then

$$
\partial_{t} \psi \leq \sum_{i, j=1}^{m}\left(\delta_{i j}-\frac{X_{i} \psi X_{j} \psi}{1+\left|\nabla_{0} \psi\right|^{2}}\right) X_{i} X_{j} \psi
$$

A function $U \in C(\tilde{G} \times[0, \infty)$ is a weak supersolution of (2.5) if

$$
\partial_{t} \psi \geq \sum_{i, j=1}^{m}\left(\delta_{i j}-\frac{X_{i} \psi X_{j} \psi}{1+\left|\nabla_{0} \psi\right|^{2}}\right) X_{i} X_{j} \psi
$$

A weak solution of (2.5) is a function $U$ which is both a weak subsolution and a weak supersolution.

As in [19], 43], in the Euclidean setting and [9] in the Heisenberg group, we have an equivalent definition of weak sub(super)solutions.

Definition 2.3. A function $u \in C(G \times[0, \infty)) \cap L^{\infty}(G \times[0, \infty))$ is a weak sub-solution of equation (2.4) if whenever $(x, t) \in G \times[0, \infty)$ for every $y X \in \mathcal{G}$ and $s \in \mathbb{R}$ and

$$
\begin{aligned}
u(\exp (y X)(x), t+s) \leq \quad u(x, t) & +\sum_{d(i)=1}^{2} p_{i} y_{i} \\
& +\frac{1}{2} \sum_{i, j=1}^{m} r_{i j} y_{i} y_{j}+q s+o\left(|y|^{2}+s^{2}\right) .
\end{aligned}
$$

for some $p \in V^{1} \oplus V_{2}, q \in \mathbb{R}$ and $R=\left(r_{i j}\right) \in \mathbb{R}^{m \times m}$ then

$$
q \leq\left\{\begin{array}{l}
\sum_{i, j=1}^{m}\left(\delta_{i j}-\frac{p_{i} p_{j}}{\left|p_{H}\right|^{2}}\right) r_{i j} \quad \text { if }\left|p_{H}\right| \neq 0 \\
\sum_{i, j=1}^{m}\left(\delta_{i j}-\eta_{i} \eta_{j}\right) r_{i j} \quad \text { for some }|\eta| \leq 1 \text { if }\left|p_{H}\right|=0
\end{array}\right.
$$

2.2.2. Generalized flow. The evolution of an initial bounded hypersuface $M_{0} \subset G$ is described in the following way: Choose a bounded function $f \in C(G)$ such that $M_{0}=\{f(x)=0\}$. We define the generalized horizontal mean curvature flow $M_{t}$ of $M_{0}$ as the level sets 
$M_{t}=\{u(x, t)=0\}$ for $u$ a weak solution of (2.4) satisfying the initial condition

$$
u(x, 0)=f(x), \text { for } x \in G .
$$

We remark explicitly that this notion of generalized flow allows for the evolution of any compact set, not necessarily an hypersurface. In order for definition to make sense one needs to show that the evolution does not depend on the choice of the defining function $f$. Lacking a suitable form of comparison principle we will not be able to prove this. however, we will establish existence and special cases of the comparison principle, leading to the basic geometric property of finite time extinction.

2.3. Preliminary results. In order to study weak solutions of (2.4) we need the subelliptic analogue of the so called sup-inf convolution as defined in [66].

Definition 2.4. For $\epsilon>$ and $u: \mathbb{R}^{n} \rightarrow \mathbb{R}$ an upper semicontinous and bounded from below function, the sup-convolution $u^{\mu}$ of $u$ is defined by

$$
u^{\mu}=\sup _{G}\left(u(y)-\frac{1}{2 \mu}\left|y^{-1} x\right|^{2 r !}\right) \forall x \in G
$$

The inf-convolution $v_{\mu}$ of $u$ is defined as

$$
u_{\mu}=\inf _{G}\left(u(y)+\frac{1}{2 \mu}\left|y^{-1} x\right|^{2 r !}\right) \forall x \in G
$$

If $x \in G$ set $|x|_{E}^{2}=x_{1}^{2}+\cdots+x_{n}^{2}$. We will say that $u$ is semiconvex if for some constant $C>0$ the function $u(x)+C|x|_{E}^{2}$ is convex in the Euclidean sense.

We use this definition of semiconvexity as in one of our proofs we will need to invoke Jensen maximum principle in the Euclidean setting.

Lemma 2.5. If $f \in C\left(R^{N}\right)$ is semi-convex and achieves a local maximum at the origin, then there exists a sequence $\left\{x^{k}\right\}_{k \in \mathbb{N}}$ converging to the origin, such that:

(i) for each $k \in N$ the function $f$ is twice differentiable in the Euclidean sense at $x^{k}$

(ii) $\left|D_{E} f\left(x^{k}\right)\right|=o(1)$ as $k \rightarrow \infty$

(iii) $D_{E}^{2} f\left(x^{k}\right) \leq o(1) I_{N}$ as $k \rightarrow \infty$

where we have denoted by $D_{E}$ and $D_{E}^{2}$ respectively the Euclidean gradient and the Euclidean Hessian, while $I_{N}$ is the identity $N \times N$ matrix. 
This lemma is a refinement by Jensen [45] of a result of Aleksandrov's. The form in which we state it is from [20, Lemma A.4].

The following lemma due to Wang plays a crucial role in our proofs:

Lemma 2.6. An upper-semicontinous function $u: G \rightarrow \mathbb{R}$ satisfies

i) $u^{\mu}$ is semiconvex and locally Lipschitz continuous with respect to $d$.

ii) $u^{\mu}$ is pointwise monotonically non decreasing in $\mu$ and converges to $u$.

iii) if $u$ is a weak subsolution of (2.4), then so is $u^{\mu}$

iv) if $u$ is continuous then $u^{\mu}$ converges to $u$ uniformly on compact sets.

Analogous results hold for the inf-convolution $u_{\mu}$. For the proof see [66, Proposition 2.3].

\section{COMPARISON PRINCIPLES}

The analysis of the generalized mean curvature flow rests on a comparison principle which roughly speaking should read as follows: If $u$ and $v$ are respectively a bounded, subsolution and supersolution of (2.4), and if $u(x, 0) \leq v(x, 0)$ for all $x \in G$ and either $u$ or $v$ are uniformly continuous at time $t=0$, then $u(x, t) \leq v(x, t)$ for all $x \in G$ and $t \geq 0$.

The sub-Riemannian geometry underlying our problem, in particular the existence of characteristic points, makes such a comparison principle much more difficult than its Euclidean counterpart (see for instance [29, Theorem 3.2]). In this section we prove two special instances of such a comparison principle, namely in Theorem 3.1 we will consider functions $u$ and $v$ satisfying more restrictive assumptions at time $t=0$ and in Theorem 3.3 we will consider only graph-like solutions in a product group $G \times \mathbb{R}$.

The main difference between the proof of our Theorem 3.1 and the corresponding Euclidean result is that the degeneration of the PDE in the Euclidean setting occurs at points where the gradient of the solution vanishes. In the subriemannian setting for the degeneration to occur it suffices that the horizontal componend of the gradient vanish. To deal with this more singular phenomena we need a fine analysis of the interplay between the stratification of the Lie algebra and the properties of super and subsolutions.

Theorem 3.1. Assume that $u$ is a bounded weak subsolution and $v$ is a bounded weak supersolution of (2.4). Suppose further

(i) For all $\left(x_{H}, x_{V}\right),\left(x_{H}, y_{V}\right) \in G u\left(x_{H}, x_{V}, 0\right) \leq v\left(x_{H}, y_{V}, 0\right)$. 
(ii) Either $u$ or $v$ is uniformly continuous when restricted to $G \times\{t=0\}$. Then $u(x, t) \leq v(x, t)$ for all $x \in G$ and $t \geq 0$.

Remark 3.2. By choosing an appropriate barrier function we use the comparison principle above it to prove the finite time extinction for compact initial data.

Proof. 1. Should the thesis fail, then for $\alpha>0$ small enough,

$$
\max _{x, t}(u(x, t)-v(x, t)-\alpha t) \geq a / 2>0 .
$$

Consequently, if we choose $\mu>0$ and sufficiently small,

$$
\max _{x, t}\left(u^{\mu}(x, t)-v_{\mu}(x, t)-\alpha t\right) \geq a / 4>0 .
$$

where the functions $u^{\mu}$ and $v_{\mu}$ denote respectively the sup and inf convolutions of $u$ and $v$, defined as in (2.6).

2. Given $\delta, \lambda>0$ define for $x \in G, y X \in \mathcal{G}$ and $t, t+s \in[0,+\infty[$

$$
\Phi(x, y, t, s)
$$

$$
\equiv u^{\mu}(y, s)-v_{\mu}(x, t)-\alpha t-\delta^{-1}\left(\left|\left(x^{-1} y\right)_{H}\right|^{4}+|s-t|^{4}\right)-\lambda\left(|x|^{2 r !}+|y|^{2 r !}+|t|^{2}+|s|^{2}\right) \text {. }
$$

We explicitly note that $\left(x^{-1} y\right)_{H}$, simply reduces to the standard Euclidean difference in the first layer $V_{1}=\mathbb{R}^{m}$. In view (3.2) we see

$$
\max _{x, y, t, s} \Phi(x, y, t, s) \geq a / 4>0 .
$$

Choose a point $(\bar{x}, \bar{y}, \bar{t}, \bar{s})$, so that

$$
\Phi(\bar{x}, \bar{y}, \bar{t}, \bar{s})=\max _{x, y, t, s} \Phi(x, y, t, s) .
$$

Then (3.3) and (3.4) together with the boundedness of $u^{\mu}$ and $v_{\mu}$, implies

$$
\lambda\left(|\bar{x}|^{2 r !}+|\bar{y}|^{2 r !}+|\bar{t}|^{2}+|\bar{s}|^{2}\right) \leq C, \quad\left|\left(\bar{x}^{-1} \bar{y}\right)_{H}\right|, \quad|\bar{s}-\bar{t}| \leq C \delta^{1 / 4} .
$$

where $C>0$ is a constant independent of $\lambda$ and $\delta$. We remark that (3.6) and the homogeneity of the gauge function implies that

$$
\left.\nabla_{0}\left(\lambda|x|^{2 r !}\right)\right|_{x=\bar{x}}=O\left(\lambda^{1 / 2 r !}\right) \text { and }\left.\nabla_{0}^{2}\left(\lambda|x|^{2 r !}\right)\right|_{x=\bar{x}}=O\left(\lambda^{1 / r !}\right),
$$

for $\lambda$ sufficiently small.

3. Arguing as in 29] and using Wang's Lemma 2.6, we deduce now that

$$
\bar{t}, \bar{s}>\sigma(\mu)=c \sqrt{\mu}
$$


and that

$$
u^{\mu} \text { is a weak subsolution }
$$

and

$$
v_{\mu} \text { is a weak supersolution. }
$$

Suppose that (3.8) does not hold, then $\bar{t}, \bar{s} \leq c \sqrt{\mu}$. Assuming $u(\cdot, 0)$ is uniformly continuous we have

0

$$
\begin{aligned}
& <a / 4 \leq \Phi(\bar{x}, \bar{y}, \bar{t}, \bar{s}) \\
& \leq u(\bar{y}, \bar{s})-v_{\mu}(\bar{x}, \bar{t}) \leq u(\bar{y}, \bar{s})-v(\bar{x}, \bar{t})+o(1) \text { as } \mu \rightarrow 0 \quad \text { (in view of Lemma 2.6) } \\
& \leq u(\bar{y}, 0)-v(\bar{x}, 0)+o(1) \text { as } \mu \rightarrow 0 \quad \text { (in view of continuity ) } \\
& =u\left(\left(\bar{y}_{H}, \bar{y}_{V}\right), 0\right)-v\left(\left(\bar{x}_{H}, \bar{x}_{V}\right), 0\right)+o(1) \text { as } \mu \rightarrow 0 \\
& \leq u\left(\left(\bar{x}_{H}, \bar{y}_{V}\right), 0\right)-v\left(\left(\bar{x}_{H}, \bar{x}_{V}\right), 0\right)+o(1) \text { as } \mu \rightarrow 0 \\
& \quad \text { and } \delta \rightarrow 0 \quad \text { (in view of the uniform continuity of } u) \\
& \leq o(1) \text { (in view of assumption (i)). }
\end{aligned}
$$

4. Next, we show that $\left|\bar{y}^{-1} \bar{x}\right|_{H}$ is bounded away from zero uniformly in $\lambda$. Using the fact that $(\bar{x}, \bar{y}, \bar{t}, \bar{s})$ is a maximum point

$$
\begin{aligned}
& u^{\mu}(y, s)-v_{\mu}(x, t)-\alpha t-\delta^{-1}\left(\left|\left(x^{-1} y\right)_{H}\right|^{4}+|s-t|^{4}\right)-\lambda\left(|x|^{2 r !}+|y|^{2 r !}+|t|^{2}+|s|^{2}\right) \\
\leq & u^{\mu}(\bar{y}, \bar{s})-v_{\mu}(\bar{x}, \bar{t})-\alpha \bar{t}-\delta^{-1}\left(\left|\left(\bar{x}^{-1} \bar{y}\right)_{H}\right|^{4}+|\bar{s}-\bar{t}|^{4}\right)-\lambda\left(|\bar{x}|^{2 r !}+|\bar{y}|^{2 r !}+|\bar{t}|^{2}+|\bar{s}|^{2}\right) .
\end{aligned}
$$

Substituting $x=\bar{x}, t=\bar{t}$, in the previous expression yields

$$
\begin{aligned}
& u^{\mu}(y, s) \leq u^{\mu}(\bar{y}, \bar{s}) \\
& \quad \begin{array}{r}
\delta^{-1}\left(\left|\left(\bar{x}^{-1} y\right)_{H}\right|^{4}-\left|\left(\bar{x}^{-1} \bar{y}\right)_{H}\right|^{4}+|s-\bar{t}|^{4}-|\bar{s}-\bar{t}|^{4}\right) \\
+\lambda\left(|y|^{2 r !}-|\bar{y}|^{2 r !}+|s|^{2}-|\bar{s}|^{2}\right) .
\end{array}
\end{aligned}
$$

Choosing $z$ such that $y=\bar{y} z=\exp (z X)(\bar{y})$ we see that $\left(\bar{x}^{-1} y\right)_{H}=$ $\left(\bar{x}^{-1} \bar{y} z\right)_{H}$. Observe that

$$
\left|\left(\bar{x}^{-1} y\right)_{H}\right|^{4}-\left|\left(\bar{x}^{-1} \bar{y}\right)_{H}\right|^{4}=\sum_{i=1}^{m} f_{i} z_{i}+\sum_{i, j=1}^{m} f_{i j} z_{i} z_{j}+o\left(|z|^{2}\right)
$$

with

$$
\begin{aligned}
& f_{i}=-4\left|\left(\bar{y}^{-1} \bar{x}\right)_{H}\right|^{2}\left(\bar{y}^{-1} \bar{x}\right)_{i} \\
& \text { and } f_{i j}=4\left(\left|\left(\bar{y}^{-1} \bar{x}\right)_{H}\right|^{2} \delta_{i j}+2\left(\bar{y}^{-1} \bar{x}\right)_{i}\left(\bar{y}^{-1} \bar{x}\right)_{j}\right) i, j=1, \cdots, m .
\end{aligned}
$$


Moreover

$$
\lambda|y|^{2 r !}-\lambda|\bar{y}|^{2 r !}=\sum_{d(i)=1}^{2} k_{i} z_{i}+\sum_{i, j=1}^{m} k_{i j} z_{i} z_{j}+o\left(|z|^{2}\right)
$$

with

$$
\begin{aligned}
\left|k_{i}\right| \leq C \lambda|\bar{y}|^{2 r !-d(i)}=O\left(\lambda^{1 / r !}\right), & \text { and } \\
& \left|k_{i j}\right| \leq C \lambda|\bar{y}|^{2 r !-2}=O\left(\lambda^{1 / r !}\right),
\end{aligned}
$$

here we have used (3.7). Substituting (3.13) - (3.16) in (3.12) we obtain

$$
\begin{aligned}
& u^{\mu}(\exp (z X)(\bar{y}), s) \\
& \leq u^{\mu}(\bar{y}, \bar{s})+\delta^{-1}\left(\sum_{i=1}^{m} f_{i} z_{i}+\sum_{i j=1}^{m} f_{i j} z_{i} z_{j}\right)+4 \delta^{-1}(\bar{s}-\bar{t})^{3}(s-\bar{s}) \\
& +2 \lambda \bar{s}(s-\bar{s})+\sum_{d(i)=1}^{2} k_{i} z_{i}+\sum_{i j=1}^{m} k_{i j} z_{i} z_{j}+o\left(|z|^{2}+|s-\bar{s}|\right) .
\end{aligned}
$$

In view of Definition 2.3 we have

$$
\begin{aligned}
4 \delta^{-1}(\bar{s}-\bar{t})^{3}+2 \lambda \bar{s}^{2} & \leq\left.\left.\sum_{i j=1}^{m}\left(\delta_{i j}-\frac{\left(k_{i}+\delta^{-1} f_{i}\right)\left(k_{j}+\delta^{-1} f_{j}\right)}{\left|k+\delta^{-1} f\right|^{2}}\right)\right|_{y=\bar{y}}\left(k_{i j}+\delta^{-1} f_{i j}\right)\right|_{y=\bar{y}} \\
& \leq 2\left|\left(k_{i j}+\delta^{-1} f_{i j}\right)\right| \\
(3.18) & \leq C\left|\left(\bar{y}^{-1} \bar{x}\right)_{H}\right|+O\left(\lambda^{1 / r !}\right) .
\end{aligned}
$$

Substituting $y=\bar{y}, s=\bar{s}$, in (3.11) yields

$$
\begin{aligned}
& v_{\mu}(x, t) \geq v_{\mu}(\bar{x}, \bar{t})-\alpha(t-\bar{t}) \\
& -\delta^{-1}\left(\left|\left(x^{-1} \bar{y}\right)_{H}\right|^{4}-\left|\left(\bar{x}^{-1} \bar{y}\right)_{H}\right|^{4}+|\bar{s}-t|^{4}-|\bar{s}-\bar{t}|^{4}\right) \\
& +\lambda\left(|\bar{y}|^{2 r !}-|y|^{2 r !}+|\bar{t}|^{2}-|t|^{2}\right) .
\end{aligned}
$$


Setting $z=\bar{x}^{-1} x$ and arguing as above we obtain

$$
\begin{aligned}
& v_{\mu}(\exp (z X)(\bar{x}), t) \\
& \geq v_{\mu}(\bar{x}, \bar{t})-\alpha(t-\bar{t})-\delta^{-1}\left(\sum_{i=1}^{m} f_{i} z_{i}+\sum_{i j=1}^{m} f_{i j} z_{i} z_{j}\right)+4 \delta^{-1}(\bar{s}-\bar{t})^{3}(t-\bar{t}) \\
& -2 \lambda \bar{t}(t-\bar{t})-\left(\sum_{d(i)=1}^{2} k_{i} z_{i}+\sum_{i j=1}^{m} k_{i j} z_{i} z_{j}\right)+o\left(|z|^{2}+|t-\bar{t}|\right) .
\end{aligned}
$$

By Definition 2.3 it follows that

$$
\begin{aligned}
& -\left(\alpha-4 \delta^{-1}(\bar{s}-\bar{t})^{3}+2 \lambda \bar{t}^{2}\right) \\
\geq & -\left.\left.\sum_{i j=1}^{m}\left(\delta_{i j}-\frac{\left(k_{i}+\delta^{-1} f_{i}\right)\left(k_{j}+\delta^{-1} f_{j}\right)}{\left|k+\delta^{-1} f\right|^{2}}\right)\right|_{x=\bar{x}}\left(k_{i j}+\delta^{-1} f_{i j}\right)\right|_{x=\bar{x}}
\end{aligned}
$$

Consequently

$$
\begin{aligned}
\alpha \leq 4 \delta^{-1}(\bar{s}-\bar{t})^{3} & -2 \lambda \bar{t}+c\left(\left|k_{i j}\right|+\delta^{-1}\left|f_{i j}\right|\right) \\
& \leq 4 \delta^{-1}(\bar{s}-\bar{t})^{3}+C \delta^{-1}\left|\left(\bar{x}^{-1} \bar{y}\right)_{H}\right|^{2}+O\left(\lambda^{1 / r !}\right)
\end{aligned}
$$

In conclusion, using (3.18) we have

$$
\alpha \leq 2 C \delta^{-1}\left|\left(\bar{x}^{-1} \bar{y}\right)_{H}\right|^{2}
$$

for $\lambda$ sufficiently small.

5. In view of Lemma 2.6 the function

$$
\Phi(x, y, t, s)+C|x, y, t, s|_{E}^{2}
$$

is convex in the Euclidean sense in a neighborhood of $(\bar{x}, \bar{y}, \bar{t}, \bar{s})$, which is a maximum point for of $\Phi(x, y, t, s)$. Using Jensen's Lemma 2.5 we see that there exists points $\left(x^{k}, y^{k}, t^{k}, s^{k}\right)$ such that

$$
\left(x^{k}, y^{k}, t^{k}, s^{k}\right) \rightarrow(\bar{x}, \bar{y}, \bar{t}, \bar{s})
$$

$\Phi, u^{\mu}, v_{\mu}$ are twice differentiable in the Euclidean $\sqrt{4}$ sense at $\left(x^{k}, y^{k}, t^{k}, s^{k}\right)$

$$
\begin{gathered}
D_{E, x, y, t, s} \Phi\left(x^{k}, y^{k}, t^{k}, s^{k}\right) \rightarrow 0, \\
D_{E, x, y, t, s}^{2} \Phi\left(x^{k}, y^{k}, t^{k}, s^{k}\right) \leq o(1) I_{2 n+2} .
\end{gathered}
$$

From (3.24) we immediately deduce that

$$
\nabla u^{\mu}\left(y^{k}, s^{k}\right) \rightarrow p+\lambda \nabla\left(|y|^{2 r !}\right)_{\mid y=\bar{y}}
$$

\footnotetext{
${ }^{4}$ We denote derivatives in the Euclidean metric with the letter $D_{E}$
} 


$$
\nabla v_{\mu}\left(x^{k}, t^{k}\right) \rightarrow p-\lambda \nabla\left(|x|^{2 r !}\right)_{\mid x=\bar{x}}
$$

where

$$
p=4 \delta^{-1}\left|\left(\bar{y}^{-1} \bar{x}\right)_{H}\right|^{2}\left(\bar{y}^{-1} \bar{x}\right)_{H} \neq 0 .
$$

Moreover

$$
\partial_{s} u^{\mu}\left(y^{k}, s^{k}\right) \rightarrow q+2 \lambda \bar{s}, \quad \partial_{t} v_{\mu}\left(x^{k}, t^{k}\right) \rightarrow q-\alpha-2 \lambda \bar{t}
$$

with

$$
q \equiv 4 \delta^{-1}|(\bar{s}-\bar{t})|^{2}(\bar{s}-\bar{t}) .
$$

On the other hand $D_{E, x, y}^{2} \Phi=A_{1}+A_{2}$ where

$$
A_{1}=D_{E, x, y}^{2}\left(u^{\mu}-v_{\mu}-\delta^{-1}\left|\left(y^{-1} x\right)_{H}\right|^{4}\right)
$$

and

$$
A_{2}=D_{E, x, y}^{2}\left(\lambda|x|^{2 r !}+\lambda|y|^{2 r !}\right)
$$

In view of (3.25) we have that at the point $\left(x^{k}, y^{k}, t^{k}, s^{k}\right)$,

$$
A_{1} \leq o(1) I_{2 n+2}-A_{2} .
$$

If we denote with $A$ the Hessian in the $x$ variable of $\left|\left(y^{-1} x\right)_{H}\right|$, then for every $w \in \mathbb{R}^{n}$

$$
\begin{aligned}
& (w, w) A_{1}\left(\begin{array}{l}
w \\
w
\end{array}\right) \\
& =(w, w)\left(\begin{array}{rr}
D_{E, y}^{2} u^{\mu}(y, s)-A & A \\
A & -D_{E, x}^{2} v_{\mu}(x, t)
\end{array}\right)\left(\begin{array}{l}
w \\
w
\end{array}\right) \\
& =<\left(D_{E, y}^{2} u^{\mu}(y, s)-D_{E, x}^{2} v_{\mu}(x, t)\right) w, w>
\end{aligned}
$$

Using (3.25) and Lemma 5.4 it follows that

$$
R^{k}-\bar{R}^{k} \leq o(1) I_{m}-\nabla^{2}\left(\lambda\left|x^{k}\right|^{2 r !}+\lambda\left|y^{k}\right|^{2 r !}\right)^{*},
$$

where

$$
R^{k}=\nabla^{2} u^{\mu}\left(y^{k}, s^{k}\right)^{*}, \quad \bar{R}^{k}=\nabla^{2} v_{\mu}\left(x^{k}, t^{k}\right)^{*} .
$$

Using Lemma 2.6 and passing to a subsequence if necessary we see that there exist $m \times m$ matrices $R, \bar{R}$ such that $R^{k} \rightarrow R, \bar{R}^{k} \rightarrow \bar{R}$ and

$$
R-\bar{R} \leq\left(\lambda|\bar{x}|^{2 r !}+\lambda|\bar{y}|^{2 r !}\right)=O\left(\lambda^{1 / r !}\right) I_{m}
$$

\footnotetext{
${ }^{5}$ We denote with $A^{*}$ the matrix $\left(A+A^{T}\right) / 2$
} 
Using the fact that $u^{\mu}$ is a subsolution and $v_{\mu}$ is a supersolution, and passing to the limit

$$
\begin{aligned}
& q+O\left(\lambda^{1 / r !}\right) \leq\left(\delta_{i j}-\frac{\left(p_{i}+O\left(\lambda^{1 / r !}\right)\right)\left(p_{j}+O\left(\lambda^{1 / r !}\right)\right)}{\left|p+O\left(\lambda^{1 / r !}\right)\right|^{2}}\right) R_{i j} \\
& \text { and } q-\alpha+O\left(\lambda^{1 / r !}\right) \geq\left(\delta_{i j}-\frac{\left(p_{i}+O\left(\lambda^{1 / r !}\right)\right)\left(p_{j}+O\left(\lambda^{1 / r !}\right)\right)}{\left|p+O\left(\lambda^{1 / r !}\right)\right|^{2}}\right) \bar{R}_{i j} .
\end{aligned}
$$

Subtracting, for $\lambda$ sufficiently small, we obtain a contradiction, and complete the proof.

Next we turn our attention to the special case of evolving graphs

$$
u(x, e, t)=e-U(x, t)
$$

in product groups of the form $\tilde{G} \times \mathbb{R}$. As we have seen, $u$ solves (2.4) if and only if $U$ solves (2.5).

Theorem 3.3. Assume that $U$ is a bounded weak subsolution and $V$ is a bounded weak supersolution of (2.5). Suppose further

(i) For all $x \in \tilde{G} U(x, 0) \leq V(x, 0)$.

(ii) Either $U$ or $V$ is uniformly continuous when restricted to

$\tilde{G} \times\{t=0\}$. Then $U(x, t) \leq V(x, t)$ for all $x \in \tilde{G}$ and $t \geq 0$. In particular, bounded weak solutions of (2.5) are unique.

Remark 3.4. For bounded domains and in the special case of the Heisenberg group this theorem follows from the results of Bieske [9]. See also the comparison principle for the Gauss curvature flow established in [36].

Proof. We follow closely the steps in the proof of Theorem 3.1 and outline only the main differences. Arguing by contradiction one easily sees that the function

$\Phi(x, y, t, s)=U^{\mu}(y, s)-V_{\mu}(x, t)-\alpha t-\frac{1}{\delta}\left(\left|y x^{-1}\right|_{E}^{4}+|s-t|^{4}\right)-\lambda\left(|x|^{2 r !}+|y|^{2 r !}+|t|^{2}+|s|^{2}\right)$,

has a strictly positive maximum at the point $(\bar{x}, \bar{y}, \bar{t}, \bar{s})$ with

$$
\begin{gathered}
\bar{t}, \bar{s} \leq c \sqrt{\mu} \\
\lambda\left(|x|^{2 r !}+|y|^{2 r !}+|t|^{2}+|s|^{2}\right) \leq C,
\end{gathered}
$$

and

$$
\left|\bar{y} \bar{x}^{-1}\right|_{E}^{4},|\bar{s}-\bar{t}|^{4} \leq C \delta
$$


Next we invoke Jensen's Lemma 2.5] and obtain a sequence of points $\left(x^{k}, y^{k}, t^{k}, s^{k}\right)$ such that (3.23), (3.24), (3.24), and (3.25) hold. In such points we obviously have

$$
\nabla_{0}^{x} \Phi=-\nabla_{0} V_{\mu}-\frac{1}{\delta} \nabla_{0}^{x}\left(\left|y x^{-1}\right|_{E}^{4}\right)-\lambda \nabla_{0}|x|^{2 r !}
$$

and

$$
\nabla_{0}^{y} \Phi=\nabla_{0} U^{\mu}-\frac{1}{\delta} \nabla_{0}^{y}\left(\left|y x^{-1}\right|_{E}^{4}\right)-\lambda \nabla_{0}|y|^{2 r !} .
$$

Next we observe that for any differentiable function $f: \tilde{G} \rightarrow \mathbb{R}$ and for any left invariant vector field $Z$ one has

$$
Z^{x} f\left(y x^{-1}\right)=-Z^{y} f\left(y x^{-1}=\left.\frac{d}{d s} f\left(y e^{-s Z} x^{-1}\right)\right|_{s=0} .\right.
$$

Similarly, if $f$ is twice differentiable and $W$ is another left invariant vector field then

$$
Z^{x} W^{x} f\left(y x^{-1}\right)=Z^{y} W^{y} f\left(y x^{-1}\right)=-Z^{x} W^{y} f\left(y x^{-1}\right)=-Z^{x} W^{y} f\left(y x^{-1}\right)
$$

We immediately deduce that

$$
\begin{aligned}
& \nabla U^{\mu}\left(y^{k}, s^{k}\right) \rightarrow p+\lambda \nabla_{0}\left(|y|^{2 r !}\right)_{\mid y=\bar{y}} \\
& \nabla V_{\mu}\left(x^{k}, t^{k}\right) \rightarrow p-\lambda \nabla_{0}\left(|x|^{2 r !}\right)_{\mid x=\bar{x}}
\end{aligned}
$$

where

$$
p=\nabla_{0}^{y}\left(\left|y x^{-1}\right|_{E}^{4}\right)
$$

Moreover

$$
\partial_{s} u^{\mu}\left(y^{k}, s^{k}\right) \rightarrow q+2 \lambda \bar{s}, \quad \partial_{t} v_{\mu}\left(x^{k}, t^{k}\right) \rightarrow q-\alpha-2 \lambda \bar{t}
$$

with

$$
q \equiv 4 \delta^{-1}|(\bar{s}-\bar{t})|^{2}(\bar{s}-\bar{t}) .
$$

Note that, unlike for the PDE (2.4), here we do not have to prove that $p \neq 0$, as $(2.5)$ does not degenerate with the vanishing of the gradient of its solution.

Using the computations above it is fairly straightforward to reproduce the argument in (3.29)-(3.30) and thus conclude the proof of the theorem.

\section{Construction of Barriers}

In this section we construct explicit bounded weak solutions of (1.3), which we later use as barrier functions in the proof of the existence theorem. 


\subsection{Self-shrinking cylinder. Let}

$$
u_{0}(x, t)=\frac{\left|x_{H}\right|^{2}}{2}+(m-1) t .
$$

This function depends only on the first layer variables and the mean curvature operator, restricted to this layer reduce to the Euclidean mean curvature operator in $\mathbb{R}^{m}$. The function $u_{0}$ satisfies (2.4) away from the characteristic set $\{0\} \times V^{2} \oplus \cdots V^{r}$ (it is actually a weak solution in all of $G$ ). The level sets $M_{t}=\left\{x: u_{0}(x, t)=\frac{R_{0}}{2}\right\}$ of this function are products of a sphere evolving by Euclidean mean curvature flow in $V_{1}$ with initial data $\partial B\left(0, R_{0}\right)$, with the higher layers $V_{2} \oplus \cdots \oplus V_{r}$. Note that the classical evolution is defined up to time $\frac{R_{0}^{2}}{2(m-1)}$. Moreover $M_{t}$ do not contain any characteristic point and constitute a self-similar flow, i.e. $M_{t}=\delta_{\lambda(t)} M_{0}$, with $\lambda=\sqrt{\frac{R_{0}^{2}}{2}-t(m-1)}$.

4.2. Coordinate planes are equilibrium solutions. Our goal here is to show that the coordinate planes $x_{i}=0, d(i)=1,2$ are minimal surfaces, i.e. their mean curvature vanishes identically outside of their characteristic set.

Remark 4.1. The result is false if $d(i)=3$ as one can easily see by examining the plane $x_{4}=0$ in the Engel group [18]. This group is best described in terms of its Lie algebra stratification $\mathcal{G}=V_{1} \oplus V_{2} \oplus V_{3}$, where the dimension of $V_{1}$ is 2 and the dimension of $V_{2}$ and $V_{3}$ is 1. The algebra has a system of generators $X_{1}, X_{2} \in V_{1}$ satisfying $\left[X_{1}, X_{2}\right]=X_{3} \in V_{2},\left[X_{1}, X_{3}\right]=X_{4} \in V_{3}$ and all the other commutators vanish. A possible representation of these vector fields in coordinates $\left(x_{1}, \cdots, x_{4}\right)$ is

$$
\begin{gathered}
X_{1}=\partial_{x_{1}}-\frac{1}{2} x_{2} \partial_{x_{3}}-\left(\frac{x_{3}}{2}+\frac{x_{1} x_{2}}{12}\right) \partial_{x_{4}}, \quad X_{2}=\partial_{x_{2}}+\frac{1}{2} x_{1} \partial_{x_{3}}+\frac{1}{12} x_{1}^{2} \partial_{x_{4}} \\
X_{3}=\partial_{x_{3}}+\frac{x_{1}}{2} \partial_{x_{4}}, \text { and } \quad X_{4}=\partial_{x_{4}} .
\end{gathered}
$$

A direct computation yields

$$
K_{0}=-\left(\left(\frac{x_{3}}{2}+\frac{x_{1} x_{2}}{12}\right)^{2}+\frac{x_{1}^{4}}{144}\right)^{-3 / 2} \frac{x_{1}^{3} x_{3}}{144},
$$

away from the characteristic points.

The starting point of our argument is the expression (2.1) for the vector fields $X_{i}, d(i)=1$ in terms of exponential coordinates

$$
X_{i}=\partial_{x_{i}}+\sum_{d(j)=1, d(h)=2} c_{i j}^{h} x_{j} \partial_{x_{h}}+\text { higher order terms . }
$$


The Campbell-Hausdorff formula implies the anti-symmetry relation $c_{i j}^{h}=-c_{j i}^{h}$. It is immediate to observe that, if $d(k)=2$ one has

$$
X_{i}\left(x_{k}\right)=\sum_{d(j)=1} c_{i j}^{k} x_{j}, \text { and } X_{i} X_{j}\left(x_{k}\right)=c_{j i}^{k}, \text { for } d(i)=d(j)=1
$$

Set $u(x)=x_{k}, d(k)=2$ then $\left|\nabla_{0} u\right|^{2} \leq C\left(x_{1}^{2}+\cdots+x_{m}^{2}\right)$ and $\left(X_{i} X_{j} u\right)^{*}=$ 0. Consequently,

$$
\sum_{i, j=1}^{m}\left(\delta_{i j}-\frac{X_{i} u_{k} X_{j} u}{\left|\nabla_{0} u\right|^{2}}\right) X_{i} X_{j} u=0
$$

if $\left|\nabla_{0} u\right| \neq 0$.

Let us explicitly note that all the barriers $u_{0}$ (as in Section 4.1), $u_{k}=x_{k}^{2}$ (with $d(k)=2$ ) we have constructed so far, satisfy the following properties

(H1) $u_{k}$ are solutions of the equation (2.4) in $\left\{x \in G|| \nabla_{0} u_{k} \mid \neq 0\right\} \times$ $(0, \infty)$.

(H2) $u_{k}$ are subcaloric (i.e. $\left.\partial_{t} u_{k} \leq \sum_{i=1}^{m} X_{i}^{2} u_{k}\right)$ in $G \times(0, \infty)$.

(H3) For every $C>0$ there exists $\tilde{C}>0$ such that if $\left|x_{H}\right|,\left|u_{k}\right| \leq C$ then $\left|\nabla_{1} u_{k}\right|+\sum_{i, j=1}^{n}\left|X_{i} X_{j} u_{k}\right| \leq \tilde{C}, d(k) \leq 2$.

4.3. Bounded barriers. Define the cut-off function $\psi:[0, \infty) \rightarrow \mathbb{R}$,

$$
\psi(s)= \begin{cases}(s-2)^{3} & \text { if } 0 \leq s \leq 2 \\ 0 & \text { if } 2 \leq s\end{cases}
$$

Note that

$$
-8 \leq \psi \leq 0, \quad \psi^{\prime} \geq 0, \quad\left|\psi^{\prime \prime}\right| \leq C_{1} \sqrt{\psi^{\prime}} \leq C_{2},
$$

Set $v_{i}(x, t)=\psi\left(u_{i}(x, t)\right)$, where $u_{i}$ are $C_{E}^{2}$ functions satisfying (H1)(H2) above.

Lemma 4.2. Assume there exists $C>0$ such that

$$
\psi^{\prime \prime}\left(u_{k}\right)\left|\nabla_{1} u_{k}\right| \leq C \text { and } \psi^{\prime}\left(u_{k}\right) \sum_{i, j=1}^{n}\left|X_{i} X_{j} u_{k}\right| \leq C .
$$

There exists $C_{0}=C_{0}\left(C_{1}, C_{2}, C\right)$ such that if we set $w_{i}^{\delta}(x, t)=v_{i}(x, t)-$ $C_{0} \sqrt{\delta}$, then for all $x \in G, t>0$ and $\epsilon>0$ sufficiently small with respect to $\delta$, one has

$$
\partial_{t} w_{k}^{\delta} \leq \sum_{i, j=1}^{n}\left(\delta_{i j}-\frac{X_{i}^{\epsilon} w_{k}^{\delta} X_{j}^{\epsilon} w_{k}^{\delta}}{\left|\nabla_{\epsilon} w_{k}^{\delta}\right|^{2}+\delta^{2}}\right) X_{i}^{\epsilon} X_{j}^{\epsilon} w_{k}^{\delta}
$$

\footnotetext{
${ }^{6}$ Here we recall that $\nabla_{1}$ denotes the full Riemannian gradient in the metric $g_{1}$.
} 
Remark 4.3. Note that in view of (H3), estimates (4.4) hold for $k=0$ with no further assumption. If $\left|x_{H}\right| \leq C$ then (4.4) hold also for $d(k)=2$

Proof. It suffices to show that

$$
\partial_{t} v_{k}-\sum_{i, j=1}^{n}\left(\delta_{i j}-\frac{X_{i}^{\epsilon} v_{k} X_{j}^{\epsilon} v_{k}}{\left|\nabla_{\epsilon} v_{k}\right|^{2}+\delta^{2}}\right) X_{i}^{\epsilon} X_{j}^{\epsilon} v_{k} \leq C_{0} \sqrt{\delta} .
$$

The left-hand side can be rewritten as

$$
\begin{aligned}
& \psi^{\prime}\left(u_{k}\right) \partial_{t} u_{k} \\
& -\sum_{i, j=1}^{n}\left(\delta_{i j}-\frac{\psi^{\prime}\left(u_{k}\right)^{2} X_{i}^{\epsilon} u_{k} X_{j}^{\epsilon} u_{k}}{\psi^{\prime}\left(u_{k}\right)^{2}\left|\nabla_{\epsilon} u_{k}\right|^{2}+\delta^{2}}\right)\left(\psi^{\prime}\left(u_{k}\right) X_{i}^{\epsilon} X_{j}^{\epsilon} u_{k}+\psi^{\prime \prime}\left(u_{k}\right) X_{i}^{\epsilon} u_{k} X_{j}^{\epsilon} u_{k}\right) \\
& =\psi^{\prime}\left(u_{k}\right) \partial_{t} u_{k}-\sum_{d(i)=d(j)=1}(\cdots)-\sum_{\substack{d(i)+d(j)>2 \\
=\psi^{\prime}\left(u_{k}\right) \partial_{t} u_{k}+S_{1}+S_{2} .}}(\cdots)
\end{aligned}
$$

Now we distinguish two cases: If $\left|\nabla_{0} u_{k}\right|=0$ then we have

$$
\psi^{\prime}\left(u_{k}\right) \partial_{t} u_{k}+S_{1}=\psi^{\prime}\left(u_{k}\right) \partial_{t} u_{k}-\sum_{i=1}^{m} X_{i}^{2} u_{k} \leq 0 .
$$

In case $\left|\nabla_{0} u_{k}\right| \neq 0$ we decompose $S_{1}$ as follows

$$
\begin{gathered}
S_{1}=-\quad \sum_{i, j=1}^{m}\left(\delta_{i j}-\frac{X_{i} u_{k} X_{j} u_{k}}{\left|\nabla_{0} u_{k}\right|^{2}}+\frac{X_{i} u_{k} X_{j} u_{k}}{\left|\nabla_{0} u_{k}\right|^{2}}\right. \\
(4.6)-\quad \frac{X_{i} u_{k} X_{j} u_{k}\left[\psi^{\prime}\left(u_{k}\right)\right]^{2}}{\left|\nabla_{0} u_{k}\right|^{2}\left[\psi^{\prime}\left(u_{k}\right)\right]^{2}+\delta^{2}}+\frac{X_{i} u_{k} X_{j} u_{k}\left[\psi^{\prime}\left(u_{k}\right)\right]^{2}}{\left|\nabla_{0} u_{k}\right|^{2}\left[\psi^{\prime}\left(u_{k}\right)\right]^{2}+\delta^{2}} \\
\left.-\quad \frac{X_{i} u_{k} X_{j} u_{k}\left[\psi^{\prime}\left(u_{k}\right)\right]^{2}}{\left|\nabla_{\epsilon} u_{k}\right|^{2}\left[\psi^{\prime}\left(u_{k}\right)\right]^{2}+\delta^{2}}\right)\left(\psi^{\prime}\left(u_{k}\right) X_{i} X_{j} u_{k}+\psi^{\prime \prime}\left(u_{k}\right) X_{i} u_{k} X_{j} u_{k}\right) \\
=S_{11}+S_{12}+S_{13} .
\end{gathered}
$$

where

$$
\begin{aligned}
& S_{11}=-\sum_{i, j=1}^{m}\left(\frac{X_{i} u_{k} X_{j} u_{k}}{\left|\nabla_{0} u_{k}\right|^{2}}-\frac{X_{i} u_{k} X_{j} u_{k}\left[\psi^{\prime}\left(u_{k}\right)\right]^{2}}{\left|\nabla_{\epsilon} u_{k}\right|^{2}\left[\psi^{\prime}\left(u_{k}\right)\right]^{2}+\delta^{2}}\right) \psi^{\prime}\left(u_{k}\right) X_{i} u_{k} X_{j} u_{k} \\
= & -\left(\frac{\delta^{2}+\psi^{\prime}\left(u_{k}\right)^{2} \epsilon^{2} \sum_{d(i)>1}\left(X_{i} u_{k}\right)^{2}}{\left|\nabla_{\epsilon} u_{k}\right|^{2} \psi^{\prime}\left(u_{k}\right)^{2}+\delta^{2}}\right) \sum_{i, j=1}^{m} \frac{X_{i} u_{k} X_{j} u_{k}}{\left|\nabla_{0} u_{k}\right|^{2}} \psi^{\prime}\left(u_{k}\right) X_{i} X_{j} u_{k} \leq 0 .
\end{aligned}
$$


Where the last indequality follows from $\psi^{\prime} \geq 0$, hypotheses (H1) and (H2) coupled with the expression

$$
\begin{aligned}
& -\sum_{i, j=1}^{m} \frac{X_{i} u_{k} X_{j} u_{k}}{\left|\nabla_{0} u_{k}\right|^{2}} X_{i} X_{j} u_{k} \\
& \quad=\sum_{i, j=1}^{m}\left(\delta_{i j}-\frac{X_{i} u_{k} X_{j} u_{k}}{\left|\nabla_{0} u_{k}\right|^{2}}\right) X_{i} X_{j} u_{k}-\sum_{i=1}^{m} X_{i}^{2} u_{k} \leq 0
\end{aligned}
$$

Next we estimate

$$
\begin{aligned}
S_{12} & =-\sum_{i, j=1}^{m}\left(\frac{X_{i} u_{k} X_{j} u_{k}}{\left|\nabla_{0} u_{k}\right|^{2}}-\frac{X_{i} u_{k} X_{j} u_{k}\left[\psi^{\prime}\left(u_{k}\right)\right]^{2}}{\left|\nabla_{0} u_{k}\right|^{2}\left[\psi^{\prime}\left(u_{k}\right)\right]^{2}+\delta^{2}}\right) \psi^{\prime \prime}\left(u_{k}\right) X_{i} u_{k} X_{j} u_{k} \\
(4.9) & =-\psi^{\prime \prime}\left(u_{k}\right) \frac{\delta^{2}\left|\nabla_{0} u_{k}\right|^{2}}{\left|\nabla_{0} u_{k}\right|^{2}\left[\psi^{\prime}\left(u_{k}\right)\right]^{2}+\delta^{2}} .
\end{aligned}
$$

In view of (4.3), if $\psi^{\prime}\left(u_{k}\right) \geq \delta$ one has

$$
S_{12} \leq \frac{\psi^{\prime \prime}\left(u_{k}\right) \delta^{2}}{\psi^{\prime}\left(u_{k}\right)} \leq C_{0} \frac{\delta^{2}}{\left|\psi^{\prime}\left(u_{k}\right)\right|^{3 / 2}} \leq C_{0} \sqrt{\delta} .
$$

In case $\psi^{\prime}\left(u_{k}\right)<\delta$ then from (H3) and (4.4) we obtain

$$
S_{12} \leq\left|\psi^{\prime \prime}\left(u_{k}\right)\right|\left|\nabla_{0} u_{k}\right|^{2} \leq C_{0} \sqrt{\delta}
$$

Here we used the fact that $\psi^{\prime \prime}\left(u_{k}\right)=0$ if $\left|u_{k}\right| \geq 2$. If we choose $\epsilon^{2} \leq \delta^{9 / 2}$ then

$$
\begin{aligned}
S_{13} & =-\sum_{i, j=1}^{m}\left(\frac{\left[\psi^{\prime}\left(u_{k}\right)\right]^{2} X_{i} u_{k} X_{j} u_{k}}{\left|\nabla_{0} u_{k}\right|^{2}\left[\psi^{\prime}\left(u_{k}\right)\right]^{2}+\delta^{2}}-\frac{X_{i} u_{k} X_{j} u_{k}\left[\psi^{\prime}\left(u_{k}\right)\right]^{2}}{\left|\nabla_{\epsilon} u_{k}\right|^{2}\left[\psi^{\prime}\left(u_{k}\right)\right]^{2}+\delta^{2}}\right) \psi^{\prime \prime}\left(u_{k}\right) X_{i} u_{k} X_{j} u_{k} \\
& =-\psi^{\prime \prime}\left(u_{k}\right) \epsilon^{2} \frac{\left[\psi^{\prime}\left(u_{k}\right)\right]^{4}\left|\nabla_{0} u_{k}\right|^{4} \sum_{d(i)>1}\left(X_{i} u_{k}\right)^{2}}{\left(\left|\nabla_{0} u_{k}\right|^{2}\left[\psi^{\prime}\left(u_{k}\right)\right]^{2}+\delta^{2}\right)\left(\left|\nabla_{\epsilon} u_{k}\right|^{2}\left[\psi^{\prime}\left(u_{k}\right)\right]^{2}+\delta^{2}\right)} \\
& \leq C_{2}^{5} C^{6} \frac{\epsilon^{2}}{\delta^{4}} \leq C_{0} \sqrt{\delta}
\end{aligned}
$$

To conclude the proof we now estimate the higher layer derivatives in $S_{2}$. Observing that $\epsilon \leq \delta^{9 / 4} \leq \sqrt{\delta}$, one has

$$
\begin{aligned}
& S_{2}=- \sum_{d(i)+d(j)>2} \psi^{\prime \prime}\left(u_{k}\right)\left(\delta_{i j}-\frac{\psi^{\prime}\left(u_{k}\right)^{2} X_{i}^{\epsilon} u_{k} X_{j}^{\epsilon} u_{k}}{\psi^{\prime}\left(u_{k}\right)^{2}\left|\nabla_{\epsilon} u_{k}\right|^{2}+\delta^{2}}\right) \\
&\left(\psi^{\prime}\left(u_{k}\right) X_{i}^{\epsilon} X_{j}^{\epsilon} u_{k}+\psi^{\prime \prime}\left(u_{k}\right) X_{i}^{\epsilon} u_{k} X_{j}^{\epsilon} u_{k}\right)=O(\epsilon)=O(\sqrt{\delta}) .
\end{aligned}
$$




\section{EXISTENCE OF WEAK SOLUTIONS}

In this section we prove the existence of weak solutions to the initial value problem for (2.4). Such solution will arise as limit of solutions of regularized parabolic equations.

For $\delta, \sigma>0$, for all $\xi \in G$ and $1 \leq i, j \leq n$ we define the coefficients of the approximating equations

$$
A_{i j}^{\epsilon, \delta}(\xi)=\left(\delta_{i j}-\frac{\xi_{i} \xi_{j}}{|\xi|^{2}+\delta}\right),
$$

and

$$
A_{i j}^{\epsilon, \delta, \sigma}(\xi)=A_{i j}^{\epsilon, \delta}(\xi)+\sigma \delta_{i j}
$$

Proposition 5.1. For any $f \in C^{\infty}(G)$ there exists a unique solution $\left.u^{\epsilon, \delta} \in C^{\infty}(G) \times(0, \infty)\right)$ of the initial value problem

$$
\begin{aligned}
\frac{\partial}{\partial t} u^{\epsilon, \delta}=\sum_{i, j=1}^{n} A_{i j}^{\epsilon, \delta}\left(\nabla_{\epsilon} u^{\epsilon, \delta}\right) X_{i}^{\epsilon} X_{j}^{\epsilon} u^{\epsilon, \delta} \text { in } x & \in G, t>0 \\
\text { and } u^{\epsilon, \delta}(x, 0) & =f(x) \text { for all } x \in G .
\end{aligned}
$$

Moreover, for all $t>0$ one has

$$
\begin{aligned}
\left\|u^{\epsilon, \delta}(\cdot, t)\right\|_{L^{\infty}(G)} & \leq\|f\|_{L^{\infty}(G)} \\
\left\|\tilde{\nabla}_{\epsilon} u^{\epsilon, \delta}(\cdot, t)\right\|_{L^{\infty}(G)} & \leq\left\|\tilde{\nabla}_{\epsilon} f\right\|_{L^{\infty}(G)} .
\end{aligned}
$$

Corollary 5.2. Let $u, f$ be as in the statement of Theorem 5.1. For any compact set $K \subset G$ there exists $C=C(K, G)>0$ such that if $0 \leq \epsilon<1$,

$$
\left\|\nabla_{\epsilon} u^{\epsilon, \delta}(\cdot, t)\right\|_{L^{\infty}(K)} \leq C\left\|\nabla_{E} f\right\|_{L^{\infty}(G)} .
$$

Ilmanen [42, page 685] shows that there exists a unique smooth solution $u^{\epsilon, \delta}$ to (5.1) satisfying the bounds

$$
\begin{aligned}
\left\|u^{\epsilon, \delta}(\cdot, t)\right\|_{L^{\infty}(G)} & \leq\|f\|_{L^{\infty}(G)} \\
\left\|\partial_{t} u^{\epsilon, \delta}(\cdot, t)\right\|_{L^{\infty}(G)} & \leq C\left\|X_{i} X_{j} f\right\|_{L^{\infty}(G)} \\
\left\|\nabla_{\epsilon} u^{\epsilon, \delta}(\cdot, t)\right\|_{L^{\infty}(G)} & \leq e^{-\lambda t}\left\|\nabla_{\epsilon} f\right\|_{L^{\infty}(G)},
\end{aligned}
$$

where $\lambda$ denotes the lowest eigenvalue for the Ricci tensor of the Riemannian metric $g_{\epsilon}$. A direct computation (see [13] for details) shows that $\lambda=-\frac{1}{\epsilon^{2}}$. As a consequence the estimates (5.2) which are uniform in $\epsilon$ do not follow immediately from (5.3). 
Proof. We follow the outline of the analogue Euclidean result proved in [29, Theorem 4.1]. For $\sigma>0$ we consider smooth solutions] $u^{\epsilon, \delta, \sigma}$ of the equation

$$
\frac{\partial}{\partial t} u^{\epsilon, \delta, \sigma}=\sum_{i, j=1}^{n} A_{i j}^{\epsilon, \delta, \sigma}\left(\nabla_{\epsilon} u^{\epsilon, \delta, \sigma}\right) X_{i}^{\epsilon} X_{j}^{\epsilon} u^{\epsilon, \delta, \sigma}
$$

with initial data $u^{\epsilon, \delta, \sigma}(x, 0)=f(x)$, for all $x \in G$. In view of the maximum principle we obtain

$$
\left\|u^{\epsilon, \delta, \sigma}(\cdot, t)\right\|_{L^{\infty}(G)} \leq\|f\|_{L^{\infty}(G)} .
$$

Since $\tilde{X}_{1}, \ldots, \tilde{X}_{n}$ commute with the left-invariant vector fields $X_{1}, \ldots, X_{n}$ then we can differentiate (5.3) along these directions and obtain the new equation

$$
\begin{aligned}
\frac{\partial}{\partial t} w=\sum_{i, j=1}^{n}\left[A_{i j}^{\epsilon, \delta, \sigma}\left(\nabla_{\epsilon} u^{\epsilon, \delta, \sigma}\right) X_{i}^{\epsilon} X_{j}^{\epsilon} w\right. & \\
& \left.+\left(\partial_{\xi_{k}} A_{i j}^{\epsilon, \delta, \sigma}\right)\left(\nabla_{\epsilon} u^{\epsilon, \delta, \sigma}\right) X_{i}^{\epsilon} X_{j}^{\epsilon} u^{\epsilon, \delta, \sigma} X_{k} w\right]
\end{aligned}
$$

where we have let $w=\tilde{X}_{i} u^{\epsilon, \delta, \sigma}$, for all $i=1, \ldots, n$. The "elliptic" maximum principle applied to (5.5) yields

$$
\left\|\tilde{\nabla}_{\epsilon} u^{\epsilon, \delta, \sigma}(\cdot, t)\right\|_{L^{\infty}(G)} \leq\left\|\tilde{\nabla}_{\epsilon} f\right\|_{L^{\infty}(G)} .
$$

Since the right invariant vector fields $\left\{\tilde{X}_{1}, \cdots, \tilde{X}_{n}\right\}$ form a basis of the tangent bundle of $G$, estimate (5.6) implies that

$$
\left\|\nabla_{\epsilon} u^{\epsilon, \delta, \sigma}(\cdot, t)\right\|_{L^{\infty}(G)} \leq C|| \tilde{\nabla}_{\epsilon} f \|_{L^{\infty}(G)},
$$

for some positive constant $C$ depending only on $G$.

As remarked in [29] the equation (5.5) satisfies coercivity conditions

$$
\left(1-\frac{M^{2}}{M^{2}+\delta}\right)|\xi|^{2} \leq \sum_{i, j=1}^{n} A_{i j}^{\epsilon, \delta, \sigma}(\xi) \xi_{i} \xi_{j} \leq 3|\xi|^{2},
$$

uniformly in $\sigma>0$ and provided $|\xi| \leq M$. Classical parabolic regularity theory (see [46]) yields estimates on all derivatives of $u^{\epsilon, \delta, \sigma}$ which are uniform in $0<\sigma<1$. To conclude the proof we use (5.4) and (5.7), Ascoli-Arzela' convergence theorem and Ilmanen's uniqueness result to

\footnotetext{
${ }^{7}$ Existence and uniqueness are guaranteed by classical parabolic theory [46]
} 
show that $u^{\epsilon, \delta, \sigma} \rightarrow u^{\epsilon, \delta}$ uniformly in the $C^{1}$ norm on compact sets as $\sigma \rightarrow 0$.

Next, we need to extend to our setting Evans and Spruck's argument in the proof of [29, Theorem 4.2]. The difficulty here is that we have two parameters rather than one. To our advantage we have the fact that estimates (5.2) are stable with respect to both $\delta \rightarrow 0$ and $\epsilon \rightarrow 0$.

Theorem 5.3. For any bounded $f \in C(G)$ there exists a viscosity solution $u \in C(G \times(0, \infty))$ of

$$
\partial_{t} u=\sum_{i, j=1}^{m} A_{i j}^{0,0}\left(\nabla_{0} u\right) X_{i} X_{j} u \quad \text { in } G \times(0, \infty) \text { and } u(x, 0)=f(x) .
$$

Let $A^{\epsilon}=\left(a_{i j}^{\epsilon}\right)$ be the matrix of coefficients of $X_{1}^{\epsilon}, \ldots, X_{n}^{\epsilon}$ in exponential coordinates, i.e. $X_{i}^{\epsilon}=\sum_{k=1}^{n} a_{i k}^{\epsilon} \partial_{x_{k}}$.

Lemma 5.4. Let $w$ be $C^{2}$ and such that at $\left(x_{0}, t_{0}\right)$ one has $D_{E}^{2} w \leq 0$ and $\nabla_{E} w=0$, then

$$
\left(X_{i}^{\epsilon} X_{j}^{\epsilon} w\right)^{*}=\frac{X_{i}^{\epsilon} T_{j}^{\epsilon} w+X_{j}^{\epsilon} X_{i}^{\epsilon} w}{2} \leq 0 .
$$

Proof. A direct computation shows that

$$
\left(X_{i}^{\epsilon} X_{j}^{\epsilon} w\right)^{*}=\sum_{l k=1}^{n} a_{i l}^{\epsilon} a_{j k}^{\epsilon} \partial_{x_{k}} \partial_{x_{l}} w
$$

Hence, for all $\eta \in \mathbb{R}^{n}$ one has

$$
\sum_{i, j=1}^{n}\left(X_{i}^{\epsilon} X_{j}^{\epsilon} w\right)^{*} \eta_{i} \eta_{j}=\sum_{l k=1}^{n}\left(D_{E}^{2} w\right)_{l k}\left(\left[A^{\epsilon}\right]^{T} \eta\right)_{l}\left(\left[A^{\epsilon}\right]^{T} \eta\right)_{k} \leq 0 .
$$

Proof of Theorem 5.3. Without loss of generality we can assume that $\nabla_{E} f$ is bounded. The general case follows as in [29, p. 659]. Let $\epsilon_{k}, \delta_{k} \rightarrow 0$ be two sequences of positive numbers such that $\epsilon_{k} / \delta_{k} \rightarrow 0$. In view of (5.2) it is possible to find a sequence (corresponding to subsequences of $\epsilon_{k}$ and $\left.\delta_{k}\right) u^{k}=u^{\epsilon_{k}, \delta_{k}}$ of smooth solutions to (5.1), with initial data $f$ and such that there exists a locally Lipschitz (with respect to the Euclidean distance) function $u$ such that $u^{k} \rightarrow u$ uniformly on compact sets. Following the argument in [29, Theorem 4.2] we first show that $u$ is a viscosity solution of (5.8) and then prove that it is constant in a set of the form $\{|x|+t \geq R\}$, with $R$ depending on $K$.

Consider $\phi \in C^{\infty}(G \times(0, \infty))$ such that $u-\phi$ has a local strict maximum point at $\left(x_{0}, t_{0}\right)$. The uniform convergence $u^{k} \rightarrow u$ implies 
that there exists a sequence of points $\left(x_{k}, t_{k}\right) \rightarrow\left(x_{o}, t_{0}\right)$ such that $u^{k}-\phi$ has a local maximum at $\left(x_{k}, t_{k}\right)$. In particular

$$
\nabla_{E} u^{k}=\nabla_{E} \phi, \partial_{t} u^{k}=\partial_{t} \phi, \text { and } D_{E}^{2}\left(u^{k}-\phi\right) \leq 0 \text { at }\left(x_{k}, t_{k}\right) .
$$

In view of Lemma 5.4 we have that at the point $\left(x_{k}, t_{k}\right)$,

$$
\begin{aligned}
\partial_{t} \phi-A_{i j}^{\epsilon_{k}, \delta_{k}} & \left(\nabla_{\epsilon_{k}} \phi\right) X_{i}^{\epsilon_{k}} X_{j}^{\epsilon_{k}} \phi \\
& \leq \partial_{t} u^{k}-A_{i j}^{\epsilon_{k}, \delta_{k}}\left(\nabla_{\epsilon_{k}} u^{k}\right) X_{i}^{\epsilon_{k}} X_{j}^{\epsilon_{k}}\left(u^{k}+\phi-u^{k}\right) \leq 0
\end{aligned}
$$

If $\nabla_{0} \phi\left(x_{0}, t_{0}\right) \neq 0$ then we simply take the limit as $k \rightarrow \infty$ in (5.9) and conclude that $u$ satisfies condition (2.6) in the definition of viscosity subsolution. If $\nabla_{0} \phi\left(x_{0}, t_{0}\right)=0$ then we set

$$
\eta^{k}=\frac{\nabla_{\epsilon_{k}} \phi\left(x_{k}, t_{k}\right)}{\sqrt{\left|\nabla_{\epsilon_{k}} \phi\left(x_{k}, t_{k}\right)\right|^{2}+\delta_{k}^{2}}}
$$

There exists $\eta \in \mathbb{R}^{n}$ such that $\eta^{k} \rightarrow \eta$. Notice that for $j=m+1, \ldots, n$ one has

$$
\begin{aligned}
&\left|\left(\eta^{k}\right)_{j}\right|=\frac{\epsilon_{k}\left|X_{j} \phi\left(x_{k}, t_{k}\right)\right|}{\sqrt{\left|\nabla_{\epsilon_{k}} \phi\left(x_{k}, t_{k}\right)\right|^{2}+\delta_{k}^{2}}} \\
& \leq \frac{\left(\epsilon_{k} / \delta_{k}\right)\left|X_{j} \phi\left(x_{k}, t_{k}\right)\right|}{\sqrt{\left(\epsilon_{k} / \delta_{k}\right)^{2} \sum_{d(i)>1}\left(X_{i} \phi\left(x_{k}, t_{k}\right)\right)^{2}+1}}
\end{aligned}
$$

Since this expression vanishes as $k \rightarrow \infty$ we have $\eta_{j}=0$ for $j=$ $m+1, \ldots, n$. The PDE (5.9) now reads as

$$
\partial_{t} \phi\left(x_{k}, t_{k}\right)-\sum_{i, j=1}^{n}\left(\delta_{i j}-\eta_{i}^{k} \eta_{j}^{j}\right) X_{i}^{\epsilon_{k}} X_{j}^{\epsilon_{k}} \phi\left(x_{k}, t_{k}\right) \leq 0
$$

then as $k \rightarrow \infty$ we obtain

$$
\partial_{t} \phi\left(x_{0}, t_{0}\right) \leq \sum_{i, j=1}^{m}\left(\delta_{i j}-\eta_{i} \eta_{k}\right) X_{i} X_{j} \phi\left(x_{0}, t_{0}\right),
$$

concluding the proof in the case in which $u-\phi$ has a local strict maximum point at $\left(x_{0}, t_{0}\right)$. If the maximum point is not strict we argue as in [29] and repeat the argument above with $\phi$ replaced by

$$
\tilde{\phi}(x, t)=\phi(x, t)+\left|x_{0}^{-1} x\right|^{2 r !}+\left|t-t_{0}\right|^{4},
$$

Using Lemma 5.4 and repeating the previous argument one can prove the analogue of (5.9) or (5.11) and from there reaching the conclusion. 
Remark 5.5. If $|\tilde{\nabla} f| \leq C$ and $u$ is a viscosity solution of the initial value problem in Theorem 5.3, then

$$
\tilde{L} i p(u)(\cdot, t)=\sup _{x \in G, h \in \mathbb{R} \text { with } h \neq 0} \frac{u(\exp (h \tilde{X})(x), t)-u(x, t)}{|h|} \leq C .
$$

Theorem 5.6. Let $G$ be a Carnot group of step two. If we assume that the function $f \in C(G)$ is constant in a neighborhood $G \backslash K$ of infinity, then any weak solution $u$ of the initial value problem (5.8) constructed as in Theorem 5.3 is constant in a set of the form $\{|x|+t \geq R\}$, with $R$ depending on $K$.

Proof. Without loss of generality we can assume that the initial data $f$ satisfies $|f| \leq 1$ in $G$ and $f(x)=0$ if $|x|>1$. Denote by $u_{i}, d(i) \leq 2$, the barrier functions constructed in Section 4 and $\psi$ the cut-off function defined in Section 4.3. For all $x \in G$ and $t>0$ set $v_{i}(x, t)=\psi\left(u_{i}(x, t)\right)$, and $w_{i}^{\delta}(x, t)=v_{i}(x, t)-C_{0} \sqrt{\delta} t$. In view of Lemma 4.2 we have that for all $x \in G, t>0$ and $\epsilon>0$ sufficiently small with respect to $\delta$, one has

$$
\partial_{t} w_{k}^{\delta} \leq \sum_{i, j=1}^{n}\left(\delta_{i j}-\frac{X_{i}^{\epsilon} w_{k}^{\delta} X_{j}^{\epsilon} w_{k}^{\delta}}{\left|\nabla_{\epsilon} w_{k}^{\delta}\right|^{2}+\delta^{2}}\right) X_{i}^{\epsilon} X_{j}^{\epsilon} w_{k}^{\delta}
$$

in the set where (4.4) holds. Note that $w_{0}^{\delta}(x, 0)=\psi\left(\left|x_{H}\right|^{2} / 2\right)=0$ for $\left|x_{H}\right| \geq 2$ and $w_{0}^{\delta}(x, 0) \leq-1$ if $\left|x_{H}\right| \leq 1$. Also, observe that for $d(k)=2$, we have $w_{k}^{\delta}(x, 0)=0$ if $x_{k}^{2} \geq 2$ and $w_{k}^{\delta}(x, 0) \leq-1$ if $x_{k}^{2} \leq 1$. Let $u^{\epsilon, \delta}$ be as in Proposition 5.1, that is a solution of the approximating equation with initial data $g$. Since $f(x) \geq w_{0}^{\delta}(x, 0)$ for all $x \in G$, then in view of the classical comparison principle for smooth solutions of quasilinear parabolic equations (see [46]) we have $u^{\epsilon, \delta}(x, t) \geq w_{0}^{\delta}(x, t)$ for all $x \in G$ and $t>0$. In view of the uniform convergence proved above, if we let $\delta, \epsilon \rightarrow 0$ we obtain $u(x, t) \geq \psi\left(\left|x_{H}\right|^{2} / 2+(m-1) t\right) \geq 0$ for $\left|x_{H}\right|^{2} / 2+(m-1) t \geq 2$. An analgous argument yields $u(x, t)=0$ in the set $\left|x_{H}\right|^{2} / 2+(m-1) t \geq 2$.

At this point we restrict our attention to the the region $A=\{x \in$ $\left.G|| x_{H} \mid \leq 2\right\}$, since we already know that $u(x, t)$ vanishes outside $A$ for every $t>0$. Note that (4.4) holds for $w_{k}^{\delta}, d(k)=2$ in the set $A$. Applying Lemma 4.2 we obtain that $w_{k}^{\delta}$ satisfies (5.13) in $A$. Since $f(x) \geq w_{k}^{\delta}(x, 0)$ for all $x \in G$, the classical maximum principle ensures that $u^{\epsilon, \delta}(x, t) \geq w_{k}^{\delta}(x, t)$, for $x \in A, t>0$. Arguing as above $u(x, t) \geq$ $\psi\left(x_{k}^{2}\right)$ for $x \in A, t>0$. In particular $u(x, t) \geq 0$ for $x \in A$ such that $x_{k}^{2} \geq 2$. Similar arguments, applied to $-u$, yields $u=0$ in the same set. In conclusion $u(x, t)=0$ for all $(x . t)$ such that $\left|x_{H}\right|^{2} / 2+(m-1) t \geq 2$, $x_{k}^{2} \geq 2, d(k)=2$. 


\section{Some GeOMETRIC PROPERTIES OF THE FLOW}

As we mentioned earlier, lacking a complete form of the comparison principle, we cannot prove that the generalized mean curvature flow defined in Section 2, does not depend on the choice of the initial data $f$, but only on its zero level set. However we can show two basic geometric properties for the flow, namely (i) separation property and (ii) show that the right invariant distance between level sets is not increasing with time.

We say that a level set $M=\{u(x)=0\}$ is cylindric if $u\left(x_{H}, x_{V}\right)$ is constant in the $x_{V}$ variables.

Proposition 6.1. Let $M_{0}, \hat{M}_{0}$ be subset of $G$ and denote by $M_{t}$ and $\hat{M}_{t}$ the corresponding generalized flows. We have

(i) If $M_{0} \subset \hat{M}_{0}$ and $\hat{M}_{t}, t \geq 0$ is cylindric, then $M_{t} \subset \hat{M}_{t}$, for all $t>0$.

(ii) For this part we consider the flows $M_{t}, \hat{M}_{t}$ arising as level set of the solutions constructed in Theorem 5.3. If we denote by $\tilde{d}(\cdot, \cdot)$ the right invariant $C C$ distance, then

$$
\tilde{d}\left(M_{0}, \hat{M}_{0}\right) \leq \tilde{d}\left(M_{t}, \hat{M}_{t}\right)
$$

for all $t>0$.

Proof. Part (i) is a direct consequence of the comparison principle. As for (ii) assume that $\tilde{d}\left(M_{0}, \hat{M}_{0}\right)>0$. We recall a result of Monti and Serra Cassano in [52], where it is proved that $\left|\tilde{\nabla}_{0} \tilde{d}\left(\cdots, M_{0}\right)\right|=$ 1 outside $M_{0}$. Thanks to this result it is immediate to construct a function $\tilde{f}$ such that $\tilde{f}=1$ on $M_{0}$, vanishes in $\left\{x \in G: \tilde{d}\left(x, M_{0}\right)>\right.$ $\left.\tilde{d}\left(M_{0}, \hat{M}_{0}\right)\right\}$ and $|\tilde{\nabla} \tilde{f}| \leq \tilde{d}\left(M_{0}, \hat{M}_{0}\right)^{-1}$. A simple modification of this construction yelds a function $f$ such that $M_{0}$ is its zero level set, $\hat{M}_{0}$ its 1 - level set and $\left|\tilde{\nabla}_{0} f\right| \leq \tilde{d}\left(M_{0}, \hat{M}_{0}\right)^{-1}$. Let us denote by $u$ the unique weak solutions to (2.4) with initial data $f$, and denote $M_{t}$ its zero level set, and $\hat{M}_{t}$ its 1 - level set. For each $t>0$ we choose points $x \in M_{t}$, $\hat{x} \in \hat{M}_{t}$ such that $\tilde{d}(x, \hat{x})=\tilde{d}\left(M_{t}, \hat{M}_{t}\right)$. Using Corollary 5.2 we have

$$
1=|u(x, t)-u(\hat{x}, t)| \leq \tilde{L} i p(u) \tilde{d}(x, \hat{x}) \leq \tilde{L} i p(g) \tilde{d}(x, \hat{x})=\frac{\tilde{d}\left(M_{t}, \hat{M}_{t}\right)}{\tilde{d}\left(M_{0}, \hat{M}_{0}\right)}
$$

concluding the proof.

We recall that the self-similar cylinder, as defined in Section 4.1, vanishes in a finite time. As a corollary we deduce that any compact set evolves within a shrinking cylinder and vanishes in a finite time. 


\section{REFERENCES}

[1] BALOGH, Z. Size of characteristic sets and functions with prescribed gradient. J. Reine Angew. Math. 564 (2003), 63-83.

[2] Balogh, Z. M., AND Rickly, M. Regularity of convex functions on Heisenberg groups. Ann. Sc. Norm. Super. Pisa Cl. Sci. (5) 2, 4 (2003), 847-868.

[3] Barles, G., And Souganidis, P. E. Convergence of approximation schemes for fully nonlinear second order equations. Asymptotic Anal. 4, 3 (1991), 271283.

[4] Barles, G., And Souganidis, P. E. A new approach to front propagation problems: theory and applications. Arch. Rational Mech. Anal. 141, 3 (1998), 237-296.

[5] Barone Adesi, V., Serra Cassano, F., and Vittone, D. The Bernstein problem for intrinsic graphs in Heisenberg groups and calibrations. Calc. Var. Partial Differential Equations 30, 1 (2007), 17-49.

[6] Bellettini, G., and Novaga, M. Minimal barriers for geometric evolutions. J. Differential Equations 139, 1 (1997), 76-103.

[7] Bellettini, G., And Novaga, M. Comparison results between minimal barriers and viscosity solutions for geometric evolutions. Ann. Scuola Norm. Sup. Pisa Cl. Sci. (4) 26, 1 (1998), 97-131.

[8] Bieske, T. On $\infty$-harmonic functions on the Heisenberg group. Comm. Partial Differential Equations 3-4, 27 (2002), 727-761.

[9] Bieske, T. Comparison principle for parabolic equations in the Heisenberg group. Electron. J. Differential Equations (2005), No. 95, 11 pp. (electronic).

[10] Bonk, M., And Capogna, L. Mean curvature flow in the Heisenberg group. Preprint.

[11] Brakke, K. A. The motion of a surface by its mean curvature, vol. 20 of Mathematical Notes. Princeton University Press, Princeton, N.J., 1978.

[12] Capogna, L., Danielli, D., Pauls, S., and Tyson, J. An introduction to the Heisenberg group and the sub-Riemannian isoperimetric problem, vol. 259 of Progress in Mathematics. Birkhäuser Verlag, Basel, 2007.

[13] Capogna, L., Pauls, S., And Tyson, J. Horizontal geometry of hypersurfaces in carnot groups. to appear in Trans. Amer. Math. Soc.

[14] Chen, Y., Giga, Y., And Goto, S. Uniqueness and existence of viscosity solutions of generalized mean curvature flow equations. J. Diff. Geom. 33, 3 (1991), 749-786.

[15] Cheng, J.-H., Hwang, J.-F., Malchiodi, A., And Yang, P. Minimal surfaces in pseudohermitian geometry. Ann. Sc. Norm. Super. Pisa Cl. Sci. (5) 4, 1 (2005), 129-177.

[16] Cheng, J.-H., Hwang, J.-F., And Yang, P. Existence and uniqueness for p-area minimizers in the Heisenberg group. Math. Ann. 337, 2 (2007), 253-293.

[17] Citti, G., And SARti, A. A cortical based model of perceptual completion in the roto-translation space. J. Math. Imaging Vision 24, 3 (2006), 307-326.

[18] Corwin, L., And Greenleaf, F. P. Representations of nilpotent Lie groups and their applications, Part I: Basic theory and examples. Cambridge Studies in Advanced Mathematics. Cambridge University Press, Cambridge, 1990.

[19] Crandall, M. G. Viscosity solutions: a primer. In Viscosity solutions and applications (Montecatini Terme, 1995), vol. 1660 of Lecture Notes in Math. Springer, Berlin, 1997, pp. 1-43. 
[20] Crandall, M. G., Ishit, H., and Lions, P. L. User's guide to viscosity solutions of second order partial differential equations. Bull. Amer. Math. Soc. (N.S.) 27, 1 (1992), 1-67.

[21] Danielli, D., Garofalo, N., and Nhieu, D.-M. Sub-Riemannian calculus on hypersurfaces in Carnot groups. Adv. Math. 215, 1 (2007), 292-378.

[22] Danielli, D., Garofalo, N., and Nhieu, D.-N. A notable family of entire intrinsic minimal graphs in the heisenberg group which are not perimeter minimizing. preprint 2006.

[23] De Giongi, E. Barriers, boundaries, motion of manifolds. Conference held at the Department of Mathematics, University of Pavia, March 18 (1994).

[24] Derridu, M. Sur un théorème de traces. Ann. Inst. Fourier (Grenoble) 22, 2 (1972), 73-83.

[25] ECKer, K. Regularity theory for mean curvature flow. Progress in Nonlinear Differential Equations and their Applications, 57. Birkhäuser Boston Inc., Boston, MA, 2004.

[26] Evans, L., And Spruck, J. Motion of level sets by mean curvature. II. Trans. Amer. Math. Soc. 330, 1 (1992), 321-332.

[27] Evans, L., AND Spruck, J. Motion of level sets by mean curvature. III. $J$. Geom. Anal. 2, 2 (1992), 121-150.

[28] Evans, L., AND Spruck, J. Motion of level sets by mean curvature. IV. J. Geom. Anal. 5, 1 (1995), 77-114.

[29] Evans, L. C., And Spruck, J. Motion of level sets by mean curvature. I. J. Diff. Geom. 33, 3 (1991), 635-681.

[30] Folland, G. B. Subelliptic estimates and function spaces on nilpotent Lie groups. Ark. Mat. 2, 13 (1975), 161-207.

[31] Folland, G. B., AND Stein, E. M. Hardy spaces on homogeneous groups. Princeton University Press, Princeton, New Jersey, 1982.

[32] Garofalo, N., And Nhieu, D.-M. Isoperimetric and Sobolev inequalities for Carnot-Carathéodory spaces and the existence of minimal surfaces. Comm. Pure Appl. Math. 49, 10 (1996), 1081-1144.

[33] Garofalo, N., and Pauls, S. The Bernstein problem in the Heisenberg group. preprint, 2003.

[34] Gromov, M. Carnot-Carathéodory spaces seen from within. In SubRiemannian Geometry, vol. 144 of Progress in Mathematics. Birkhäuser, Basel, 1996, pp. 79-323.

[35] Gromov, M. Metric structures for Riemannian and non-Riemannian spaces. Birkhäuser Boston Inc., Boston, MA, 1999. Edited by J. LaFontaine and P. Pansu. Based on the 1981 French original, Translated from the French by Sean Michael Bates.

[36] HALLER, E. Generalized subriemannian gauss curvature flow in carnot groups. Ph.D. Dissertation, University of Arkansas (2008).

[37] Hladky, R., AND PAuls, S. D. A disocclusion algorithm based on a model of the visual cortex. In preparation.

[38] Hladky, R., and Pauls, S. D. Minimal surfaces in the Roto-translation group with applications to a neurobiological image completion model,. Preprint.

[39] Hladky, R. K., And Pauls, S. D. Constant mean curvature surfaces in sub-riemannian geometry. to appear in Jour. Diff. Geom. 
[40] Hoffman, W. The visual cortex is a contact bundle. Appl. Math. Comput. 32, 2-3 (1989), 137-167.

[41] Hörmander, L. Hypoelliptic second order differential equations. Acta Math., 119 (1967), 147-171.

[42] Ilmanen, T. Generalized flow of sets by mean curvature on a manifold. Indiana Univ. Math. J. 41, 3 (1992), 671-705.

[43] IsHII, H. Viscosity solutions of nonlinear partial differential equations [translation of Sūgaku 46 (1994), no. 2, 144-157; MR1303774 (95j:49002)]. Sugaku Expositions 9, 2 (1996), 135-152. Sugaku Expositions.

[44] IshiI, H., AND Souganidis, P. Generalized motion of noncompact hypersurfaces with velocity having arbitrary growth on the curvature tensor. Tohoku Math. J. (2) 47, 2 (1995), 227-250.

[45] Jensen, R. The maximum principle for viscosity solutions of fully nonlinear second order partial differential equations. Arch. Rational Mech. Anal. 101, 1 (1988), 1-27.

[46] Ladyženskaja, O. A., Solonnikov, V. A., and Ural'ceva, N. N. Linear and quasilinear equations of parabolic type. Translated from the Russian by S. Smith. Translations of Mathematical Monographs, Vol. 23. American Mathematical Society, Providence, R.I., 1967.

[47] Lu, G., Manfredi, J. J., And Stroffolini, B. Convex functions on the Heisenberg group. Calc. Var. Partial Differential Equations 19, 1 (2004), 1-22.

[48] Magnani, V. Characteristic points, rectifiability and perimeter measure on stratified groups. J. Eur. Math. Soc. (JEMS) 8, 4 (2006), 585-609.

[49] Magnani, V. Lipschitz continuity, Aleksandrov theorem, and characterizations for H-convex functions. Math. Ann. 334 (2006), 199-233.

[50] Mitchell, J. On Carnot-Carathéodory metrics. J. Differential Geom. 21, 1 (1985), 35-45.

[51] Montgomery, R. A tour of sub-Riemannian geometries, their geodesics and applications. No. 91 in Mathematical Surveys and Monographs. American Mathematical Society, 2002.

[52] Monti, R., And Serra Cassano, F. Surface measures in CarnotCarathéodory spaces. Calc. Var. Partial Differential Equations 13, 3 (2001), 339-376.

[53] Nagel, A., Stein, E. M., And Wainger, S. Balls and metrics defined by vector fields. I. Basic properties. Acta Math. 155, 1-2 (1985), 103-147.

[54] NI, Y. Sub-Riemannian constant mean curvature surfaces in the Heisenberg group as limits. Preprint, 2005.

[55] Osher, S., And Sethian, J. A. Fronts propagating with curvature-dependent speed: algorithms based on Hamilton-Jacobi formulations. J. Comput. Phys. 79, 1 (1988), 12-49.

[56] Pauls, S. D. Minimal surfaces in the Heisenberg group. Geom. Dedicata 104 (2004), 201-231.

[57] Peтiтot, J. The neurogeometry of pinwheels as a sub-Riemannian contact structure. J. Physiology 97 (2003), 265-309.

[58] Petitot, J., and Tondut, Y. Vers une neuro-geometrie. fibrations corticales, structures de contact et contours subjectifs modaux. Mathematiques, Informatique et Sciences Humaine, EHESS, Paris 145 (1998), 5-101. 
[59] Ritoré, M., and Rosales, C. Rotationally invariant hypersurfaces with constant mean curvature in the Heisenberg group $\mathbb{H}^{n}$. J. Geom. Anal. 16, 4 (2006), 703-720.

[60] Rothschild, L. P., And Stein, E. M. Hypoelliptic differential operators and nilpotent groups. Acta Math. 137, 3-4 (1976), 247-320.

[61] Sarti, A., Citti, G., And Manfredini, M. From neural oscillations to variational problems in the visual cortex. Journal of Physiology 97 (2003), 379-385.

[62] Selby, C. seminar at the meeting "Geometric analysis and applications" University of Illinois, Urbana-Champaign, July 12-15, 2006.

[63] Soner, H. M., And Touzi, N. A stochastic representation for the level set equations. Comm. Partial Differential Equations 27, 9-10 (2002), 2031-2053.

[64] Stein, E. M. Harmonic analysis: real-variable methods, orthogonality, and oscillatory integrals, vol. 43 of Princeton Mathematical Series. Princeton University Press, Princeton, NJ, 1993. With the assistance of Timothy S. Murphy, Monographs in Harmonic Analysis, III.

[65] WANG, C.-Y. The aronsson equation for absolute minimizers of l-infinity functionals associated with vector fields satisfying hormanders condition. Transactions of American Mathematical Society. to appear.

[66] Wang, C. Y. Viscosity convex functions on Carnot groups. Proc. Amer. Math. Soc. 133, 4 (2005), 1247-1253 (electronic).

[67] ZHu, X. P. Lectures on mean curvature flows, vol. 32 of AMS/IP Studies in Advanced Mathematics. American Mathematical Society, Providence, RI, 2002.

Department of Mathematical Sciences, University of Arkansas, FayetTEVILLE, AR 72701

E-mail address: lcapogna@uark.edu

Dipartimento di Matematica, Piazza Porta S. Donato 5, 40126 Bologna, ITALY

E-mail address: citti@dm.unibo.it 